\title{
Carleson measures for analytic Besov spaces
}

Nicola Arcozzi, Richard Rochberg and Eric Sawyer

\begin{abstract}
We characterize Carleson measures for the analytic Besov spaces. The problem is first reduced to a discrete question involving measures on trees which is then solved. Applications are given to multipliers for the Besov spaces and to the determination of interpolating sequences. The discrete theorem is also applied to analysis of function space on trees.
\end{abstract}

\section{Introduction and summary.}

Our starting point is an attempt to get a better understanding of Carleson measures for Besov spaces of holomorphic functions. To do this we first reduce to a discrete model problem which turns out to be the question of characterizing those measures on trees for which a discrete Hardy's inequality holds. We give solution to that problem, Theorem 3, and use that result in obtaining our characterization of Carleson measures on Besov spaces, Theorem 1.

Carleson measures arise in many questions involving analysis in function spaces and we consider two such questions here. We look at multipliers. That is, given a space, $X$, of functions on a set $Y$, we want to describe those functions $f$ for which the map of multiplication by $f$ is a continuous map of $X$ to itself. We show that the known results for the classical Besov spaces extend easily to the weighted Besov spaces we consider, Theorem

2000 Mathematics Subject Classification: 30H05, 46E35, 46 E39.

Keywords: Carleson measures, discrete Sobolev inequalities. 
15. We also find that these results have a satisfyingly exact analog for our spaces of functions on trees, Theorem 26.

The other problem we consider is of describing interpolating sequences That is, we want to characterize those sequences $\left\{y_{i}\right\}$ in $Y$ with the property that, as $f$ ranges over $X$, the set of sequences of values, $\left\{f\left(y_{i}\right)\right\}$ ranges over the space of all sequences which satisfy a natural growth condition. We are able to give a complete characterization of interpolating sequences in the discrete model, Theorem 26. However we do not yet see how to extend our results fully to the Besov spaces; in that context we offer only partial results, Theorems 31 and 33 .

To describe our results further we introduce some notation and terminology.

Let $\mathbb{D}$ be the unit disk in the complex plane, $z=x+i y$, and $m(d z)=$ $\pi^{-1} d x d y$ the normalized area element on $\mathbb{D}$.

For $\rho \geq 0$, a positive Borel measurable weight function on $\mathbb{D}, f: \mathbb{D} \longrightarrow$ $\mathbb{C}$ holomorphic in $\mathbb{D}$, and $1<p<\infty$, set

$$
\|f\|_{B_{p}(\rho)}^{* p}=\int_{\mathbb{D}}\left|\left(1-|z|^{2}\right) f^{\prime}(z)\right|^{p} \rho(z) \frac{m(d z)}{\left(1-|z|^{2}\right)^{2}} .
$$

The weighted Besov space $B_{p}(\rho)$ with weight $\rho$ is the space of those holomorphic functions $f$ for which $\|f\|_{B_{p}(\rho)}^{*}$ is finite. $B_{p}(\rho)$ is a Banach space under the norm

$$
\|f\|_{B_{p}(\rho)}=|f(0)|+\|f\|_{B_{p}(\rho)}^{*} .
$$

(That fact actually requires some conditions on $\rho$.However there is certainly no problem for the class of admissible weights which we introduce in a moment.) The spaces $B_{p}(\rho)$ for $\rho \equiv 1$ are the usual analytic Besov spaces and we denote them $B_{p} . B_{2}$ is the Dirichlet space and the more generally spaces $B_{2}(\rho)$ are sometimes also called Dirichlet spaces. We will study Carleson measures for $B_{p}(\rho)$; that is, given indices $p$ and $q$, and a weight $\rho$, we wish to describe measures $\mu$ such that Id, the natural identity inclusion of $B_{p}(\rho)$ into $L^{q}(\mu)$, is continuous.

For $a \in \mathbb{D}$ let

$$
S(a)=\left\{z \in \mathbb{D}: 1-|z| \leq 1-|a|,\left|\frac{\arg (a \bar{z})}{2 \pi}\right| \leq \frac{1-|a|}{2}\right\}
$$

be the Carleson box (or square) with vertex at $a$. We will also use the heightened box

$$
\widetilde{S}(a)=\left\{z \in \mathbb{D}: 1-|z| \leq 2(1-|a|),\left|\frac{\arg (a \bar{z})}{2 \pi}\right| \leq \frac{1-|a|}{2}\right\}
$$


We sometimes find it convenient to think of $\mathbb{D}$ with its hyperbolic geometry. Let $m_{h}(d z)=\left(1-|z|^{2}\right)^{-2} m(d z)$ be the hyperbolic area element. Set $\delta f(z)=\left(1-|z|^{2}\right)\left|f^{\prime}(z)\right| ; \delta f$ measures the infinitesimal distortion of $f$ regarded as a map from $\mathbb{D}$ with the hyperbolic metric to $\mathbb{C}$ with the Euclidean metric. With this notation, $B_{p}(\rho)$ consists of holomorphic functions for which $\delta f \in L^{p}\left(\rho m_{h}\right)$.

Our methods require that $\rho$ satisfy certain smoothness and growth conditions. Given $p, 1<p<\infty$, we say that $\rho$ is $p$-admissible, or, simply, admissible, if both:

i) $\rho$ is regular, i.e., there exist $\varepsilon>0, C>0$ such that $\rho\left(z_{1}\right) \leq C \rho\left(z_{2}\right)$ whenever $z_{1}$ and $z_{2}$ are within hyperbolic distance $\varepsilon$. Equivalently, there are $\delta<1, C^{\prime}>0$ so that $\rho\left(z_{1}\right) \leq C^{\prime} \rho\left(z_{2}\right)$ whenever

$$
\left|\frac{z_{1}-z_{2}}{1-z_{1} z_{2}}\right| \leq \delta<1
$$

ii) The weight $\rho_{p}(z)=\left(1-|z|^{2}\right)^{p-2} \rho(z)$ satisfies the Bekollé-Bonami $\mathcal{B}_{p}$ condition $([\mathrm{BB}],[\mathrm{Bek}])$ : There is a $C(\rho, p)$ so that for all $a \in \mathbb{D}$.

$$
\begin{gathered}
\left(\int_{S(a)} \rho_{p}(z) m(d z)\right)\left(\int_{S(a)} \rho_{p}(z)^{1-p^{\prime}} m(d z)\right)^{1 /\left(p^{\prime}-1\right)} \\
\leq C(\rho, p) m(S(a))^{p}
\end{gathered}
$$

(Here and throughout $p^{-1}+p^{-1}=q^{-1}+q^{-1}=1$.) This condition allows us to identify the dual space of $B_{p}(\rho)$ with $B_{p^{\prime}}\left(\rho^{1-p^{\prime}}\right)$.

For instance, $\rho \equiv 1$ is admissible. More generally, it is easy to check that $\rho(z)=\left(1-|z|^{2}\right)^{\alpha}$ is $p$-admissible if and only if $1-p<\alpha<1$. The interesting range is $0 \leq \alpha<1$.

When we prove that a family of inequalities $\mathbf{A}$ imply an inequality $\mathbf{B}$, we say that the constants appearing in $\mathbf{B}$ are quantitative if they only depend on the constants that appear in $\mathbf{A}$, on $p, q$ and, eventually, on the dimension.

We will prove the following

Theorem 1. Suppose $1<p \leq q<\infty$ and that $\rho$ is a $p$-admissible weight. For a positive Borel measure $\mu$ on $\mathbb{D}$ the following are equivalent:

i) There is a $C(\mu)>0$ such that

$$
\|f\|_{L^{q}(\mu)} \leq C(\mu)\|f\|_{B_{p}(\rho)} .
$$


ii) There is a $C_{1}(\mu)>0$, so that for all $a \in \mathbb{D}$

$$
\left(\int_{\widetilde{S}(a)} \rho(z)^{-p^{\prime} / p}(\mu(S(z) \cap S(a)))^{p^{\prime}} m_{h}(d z)\right)^{q^{\prime} / p^{\prime}} \leq C_{1}(\mu) \mu(S(a)) .
$$

The constants are quantitative.

In Section 5.5 we give an alternative formulation of (2) which emphasizes its conformal invariance. Under the more restrictive assumption that $p<q$, however, the characterization is much simpler and, as we shall see later, analogous to the characterization of the Carleson measures for the Hardy spaces.

Theorem 2. If $1<p<q<\infty$ and $\rho$ is a $p$-admissible weight, then (2) holds if and only if

$$
\mu(S(a))^{1 / q} \leq C\left(\int_{[0, a]} \rho(w)^{1-p^{\prime}} \frac{|d w|}{1-|w|^{2}}\right)^{-1 / p^{\prime}}
$$

The constants are quantitative.

In the right hand side $[0, a]$ denotes the segment between 0 and $a$. We will see, later, that, for $p=q$, (4) is weaker than (3).

We say that a positive measure $\mu$ on $\mathbb{D}$ is a Carleson measure for $\left(B_{p}(\rho), q\right)$ if it satisfies the inequality $(2)$. When $p=q$, we will simply say that $\mu$ is Carleson for $B_{p}(\rho)$. We will see in Section 5.5 that (2) has an equivalent conformally invariant formulation.

The classical result of Carleson [Car] for the Hardy space corresponds to the weight $\rho(z)=1-|z|^{2}$ which is not 2 -admissible. The first result involving admissible weights is due to Stegenga [Ste]. He considered $p=$ $q=2$ and showed, among other thing, that for the Dirichlet space, i.e. for $\rho \equiv 1$, the Carleson measures could be characterized by a geometric condition involving the logarithmic capacity of subsets of $\partial \mathbb{D}$. Stegenga's theorem was generalized by I. Verbitsky [Ve] to $1<p=q<\infty$, again by using appropriate capacities. Unfortunately, that paper did not have the attention it deserved, and the result was recently rediscovered by J. Wang [Wang]. More recent work in a similar vein is in [Wu], [CO1], and [CO2]. In [KS], Kerman and Sawyer characterized the Carleson measures for $B_{2}(\rho)$ for certain radial weights $\rho$. The conditions in [KS] do not involve capacities and are of a different form than those in Theorem 1. The two sets of results are compared in detail in Section 5 . 
Theorem 1 goes further in that it also applies to weights that are not radial and covers $p$ in the range $(1, \infty)$. Perhaps the most interesting aspect of Theorem 1 is the form of condition (3) which characterizes the Carleson measures. The condition seems easier to work with than the earlier conditions; in particular, it seems much easier to verify than the capacitary conditions of Stegenga, Verbitsky, Wang, and Wu.

The proof of Theorem 1 is based on a discretization procedure and on the solution of the resulting discrete problem which involves measures on trees.

Let $T$ be a tree, i.e., a connected, loopless graph, that we do not assume to be locally finite; see Section 3 for complete definitions and notation. Let $o \in T$ be a fixed vertex, the root of $T$. There is a partial order on $T$ defined by: $x \leq y, x, y \in T$, if $x \in[o, y]$, the geodesic joining $o$ and $y$. Let $\varphi: T \longrightarrow \mathbb{C}$. We define $\mathcal{I} \varphi$, the primitive of $\varphi$ with respect to $o$, by

$$
\mathcal{I} \varphi(x)=\sum_{o}^{x} \varphi(y)=\sum_{y \in[o, x]} \varphi(y)
$$

A weight $\rho$ on $T$ is a positive function on $T$.

For $x \in T$, let $S(x)=\{y \in T: y \geq x\} . S(x)$ is the Carleson square with vertex $x$ or the shadow of $x$.

Theorem 3. Let $1<p \leq q<\infty$ and let $\rho$ be a weight on $T$. For a nonnegative function $\mu$ on $T$, the following are equivalent:

1) For some constant $C(\mu)>0$ and all functions $\varphi$

$$
\left(\sum_{x \in T}|\mathcal{I} \varphi(x)|{ }^{q} \mu(x)\right)^{1 / q} \leq C(\mu)\left(\sum_{x \in T}|\varphi(x)|^{p} \rho(x)\right)^{1 / p} .
$$

2) For some $C(\mu)>0$ and all $r \in T$

$$
\left(\sum_{x \in S(r)}\left(\sum_{y \in S(x)} \mu(y)\right)^{p^{\prime}} \rho(x)^{1-p^{\prime}}\right)^{q^{\prime} / p^{\prime}} \leq C(\mu) \sum_{x \in S(r)} \mu(x) .
$$

The constants are quantitative.

When $p<q$, we again have a much simpler condition. 
Theorem 4. With the assumptions of Theorem 3 and $p<q$, (5) holds if and only if, for some $C(\mu)>0$ and all $r \in T$,

$$
\left(\sum_{x \in S(r)} \mu(x)\right)^{1 / q} \leq C\left(\sum_{0}^{r} \rho(x)^{1-p^{\prime}}\right)^{-1 / p^{\prime}} .
$$

The constants are quantitative.

In analogy with the continuous case, we say that a measure satisfying $(5)$ is a Carleson measure for $(\mathcal{I}, \rho, p, q)$.

In fact, inequality (5) is a weighted Hardy inequality on the tree $T$. For more on weighted Hardy inequalities see the survey [Muck] and the articles [Saw], [SW].

Given Theorem 3, the proof of Theorem 1 is rather simple. To prove that the geometric condition (3) forces $\mu$ to be Carleson we discretize the holomorphic functions and use Theorem 3. Here is an outline of that argument. Consider a dyadic Whitney decomposition of $\mathbb{D}$. Namely, for integer $n \geq 0,1 \leq m \leq 2^{n}$, let

$\Delta_{n, m}=\left\{z \in \mathbb{D}: 2^{-n-1} \leq 1-|z| \leq 2^{-n},\left|\frac{\arg (z)}{2 \pi}-\frac{m-1 / 2}{2^{n}}\right| \leq 2^{-(n+1)}\right\}$.

It is natural to consider the Whitney squares as indexed by the vertices of a dyadic tree, $T_{2}$. Thus the vertices of $T_{2}$ are

$$
\left\{\alpha: \alpha=(n, m), n \geq 0 \text { and } 1 \leq m \leq 2^{n}, m, n \in \mathbb{N}\right\}
$$

and we say that there is an edge between $(n, m),\left(n^{\prime}, m^{\prime}\right)$ if $\Delta_{(n, m)}$ and $\Delta_{\left(n^{\prime}, m^{\prime}\right)}$ share an arc of a circle. The root of $T_{2}$ is, by definition, $(0,1)$. Here and throughout we will abuse notation and, when convenient, identify the vertices of such a tree with the sets for which they are indices. Here we identify $\alpha$ and $\Delta_{\alpha}$.

If $f \in B_{p}(\rho)$ and $\alpha \in T_{2}$, let $\varphi(\alpha)=\left(1-\left|z_{\alpha}\right|^{2}\right) f^{\prime}\left(z_{\alpha}\right)$, where $z_{\alpha} \in \alpha$ is chosen as to maximize $|f(z)|$ in $\alpha$. Then, by the assumptions on $\rho$ and, essentially, the mean value property,

$$
\|f\|_{B_{p}(\rho)} \geq \frac{1}{C} \sum_{\alpha \in T_{2}}|\varphi(\alpha)|^{p} \rho\left(z_{\alpha}\right)
$$

and

$$
\left|f\left(z_{\alpha}\right)\right|=\left|f(0)+\int_{0}^{z_{\alpha}}\left(1-|\xi|^{2}\right) f^{\prime}(\xi) \frac{d \xi}{\left(1-|\xi|^{2}\right)}\right| \leq C \sum_{\beta=o}^{\alpha} \varphi(\beta) .
$$


Thus

$$
\|f\|_{L^{q}(\mu)} \leq C\left(\sum_{\alpha \in T_{2}}|\mathcal{I}(\alpha)|^{q} \mu(\alpha)\right)^{1 / q} .
$$

It then follows easily from Theorem 3 that a positive measure $\mu$ on $\mathbb{D}$ is Carleson for $\left(B_{p}(\rho), q\right)$ if $(6)$ is satisfied. In Section 5 we give a show that (6) is equivalent to (3). To prove the converse, we test an inequality which is dual to (2) on weighted characteristic functions of Carleson boxes in $\mathbb{D}$.

The proof of Theorem 3 is more technical and is based on a good- $\lambda$ type argument. A crucial fact in the proof is that there is no issue of cancellation in the theorem. Hence it suffices to prove the theorem for positive $\varphi$. For positive $\varphi$ the monotonicity properties of $\mathcal{I} \varphi$ can be used decisively.

In fact there is no loss of information in the passage to this discrete model problem. Given a measure $\mu$ on $\mathbb{D}$ and an admissible weight $\rho$; $\mu$ can be regarded as a function on $T_{2}$ and $\rho$ can be defined on $T_{2}$ by $\rho(\alpha)=\rho\left(\Delta_{\alpha}\right)$, where $\xi_{\alpha}$ is, say, the center of the Whitney box $\alpha$. We then have

Proposition 5. For $1<p \leq q<\infty, \mu$ is a Carleson measure for $\left(B_{p}(\rho), q\right)$ if and only if it is a Carleson measure for $(\mathcal{I}, \rho, p, q)$.

Proposition 5 might appear surprising because some structure was discarded in the discretization process. For instance, neither $\varphi$ nor $\mathcal{I} \varphi$ are required to satisfy any variant of the mean value property. That the proposition holds nevertheless seems linked to the $\mathcal{B}_{p}$ condition. For instance, the $\mathcal{B}_{2}$ condition does not hold for $\rho(z)=1-|z|^{2}$ and we will see that both Theorem 1 and Proposition 5 fail for the weighted Dirichlet space $B_{2}\left(1-|z|^{2}\right)$ (= the Hardy space, $\left.H^{2}\right)$.

The article is structured as follows. In Section 2 we discuss some facts about Bergman spaces. We show how the discretization procedure works in the simpler context of Bergman spaces by providing a proof of Luecking's characterization of the Carleson measures for the weighted Bergman spaces [L]. Section 3 is dedicated to the proof of Theorems 3 and 4 and to a closer analysis of some classes of Carleson measures on trees. We also show that a simple condition on a measure, analogous to Carleson's condition in $H^{2}$, is not generally sufficient for the measure to be a Carleson measure on a tree. In Section 4 we prove Theorems 1 and 2, modulo the equivalence between (3) and (6), and we also characterize multipliers for $B_{p}(\rho)$. The equivalence of (3) and (6) is Proposition 16 in Section 5. In Section 5 we also compare several characterizations for the Carleson measures for Dirichlet spaces and give concrete conditions for some weights to be admissible. That section 
also contains an analysis of the relation between our results and those in [KS]. In Corollary 23 we show that the two conditions can be viewed as endpoints of a scale of equivalent characterizations of Carleson measures. Also, we show how Carleson measures can be defined in a conformally invariant fashion.

In Section 6 we characterize multipliers and interpolating sequences for certain function spaces on trees. Some partial results for interpolating sequences for the analytic Besov spaces are discussed in Section 7.

Theorem 3 does not require that $\rho$ be admissible and hence it applies if $\rho(z)$ is (a discrete version of) $\left(1-|z|^{2}\right)$. However the conclusion obtained is not a discrete analog of Carleson's pioneering theorem for $B_{2}(\rho)=H^{2}(\partial \mathbb{D})$. We pursue this further in Section 8 , where we show that if we only require (5) to hold for discrete harmonic functions (i.e. those which satisfy a mean value equality) then the resulting class of measures is characterized by a single box condition analogous to the classical one, Theorem 37.

A more detailed outline of the contents is given at the beginning of each section.

\section{Carleson measures on Bergman spaces.}

The Carleson measures for weighted Bergman spaces were characterized by Luecking [L]. In Theorem 7 we give an alternative proof to some of those results. This lets us display the basic approach used in proving Theorem 1 in a more straightforward context. Some of the background material on Bergman spaces from this section is used later, however the results in other sections are independent of Theorem 7.

Let $1<p<\infty$ be fixed and let $\rho$ be a weight on $\mathbb{D}$. The Bergman space $A_{p}(\rho)$ is the space of those functions $f$ that are holomorphic in $\mathbb{D}$ and such that

$$
\|f\|_{A_{p}(\rho)}^{p}=\int_{\mathbb{D}}|f(z)|^{p} \rho(z) m(d z)
$$

is finite. Define, for $f, g \in A_{2} \equiv A_{2}(1)$,

$$
\langle f, g\rangle_{A_{2}}=\int_{\mathbb{D}} f(z) \overline{g(z)} m(d z) .
$$

Let $A_{p}(\rho)^{*}$ be the dual space of $A_{p}(\rho)$. We identify $g \in A_{p^{\prime}}\left(\rho^{1-p^{\prime}}\right)$ with the functional on $A_{p}(\rho)$

$$
\Lambda_{g}: f \longmapsto\langle f, g\rangle_{A_{2}}
$$


By Hölder's inequality we have that $A_{p}^{*}(\rho) \subseteq A_{p^{\prime}}\left(\rho^{1-p^{\prime}}\right)$. Condition (1) allows us to reverse the inclusion.

Theorem 6 (Bekollé-Bonami $[\mathrm{BB}],[\mathrm{Bek}])$. If the weight $\rho$ satisfies (1) then $g \longmapsto \Lambda_{g}$, where $\Lambda_{g}$ is defined in (9) is an isomorphism of $A_{p^{\prime}}\left(\rho^{1-p^{\prime}}\right)$ onto $A_{p}^{*}(\rho)$.

(Actually, the papers mentioned have results on the boundedness of the Bergman projection on certain weighted Lebesgue spaces. However the theorem stated follows easily; see [L, Theorem 2.1] for a detailed argument.)

In many respects, Theorem 6 allows us to work with spaces of analytic functions "as if" they were $L^{p}$ spaces.

We will see, now, how the determination of the Carleson measures for the Bergman spaces can be reduced to a discrete inequality, one which happens to be trivial in this case. Recall that a measure $\mu$ on $\mathbb{D}$ is Carleson for $A_{p}(\rho)$ if, for all $f \in A_{p}(\rho)$,

$$
\int_{\mathbb{D}}|f(z)|^{p} \mu(d z) \leq C(\mu) \int_{\mathbb{D}}|f(z)|^{p} m(d z) .
$$

Let $N$ be a fixed, positive integer, to be chosen later. For each integer $n \geq 0$ and $1 \leq m \leq N 2^{n}$, let

$\Delta_{n, m}=\left\{z \in \mathbb{D}: 2^{-n-1} \leq 1-|z| \leq 2^{-n},\left|\frac{\arg (z)}{2 \pi}-\frac{m-1 / 2}{2^{n}}\right| \leq N^{-1} 2^{-n}\right\}$

and

$$
T_{2}=\left\{\alpha=(n, m) \in \mathbb{N} \times \mathbb{N}: n \geq 0,1 \leq m \leq N 2^{n}\right\} .
$$

The regions $\Delta_{\alpha}$ form a Whitney decomposition of $\mathbb{D}$; i.e., in terms of the Euclidean diameter and distance

$$
\operatorname{diam}\left(\Delta_{\alpha}\right) \sim \operatorname{dist}\left(\Delta_{\alpha}, \partial \mathbb{D}\right)
$$

From the viewpoint of hyperbolic geometry, the regions $\Delta_{\alpha}$ all have comparable diameter and all have comparable volume. With harmless abuse, we identify $\Delta_{\alpha}$ and $\alpha$.

Let $\rho$ be an admissible weight and set

$$
\lambda(\alpha)=\int_{\alpha} \rho(z) m(d z) .
$$


Theorem 7 (Luecking $[\mathrm{L}]$ ). A measure $\mu$ on $\mathbb{D}$ is a Carleson measure for $A_{p}(\rho)$ if and only if, for some $C>0$ and for all $\alpha \in T_{2}$,

$$
\mu(\alpha) \leq C \lambda(\alpha)
$$

The constants are quantitative.

Suppose that (10) holds and let $f \in A_{p}(\rho)$. Because $f$ is continuous we can pick $z_{\alpha} \in \alpha$ so that

$$
\int_{\mathbb{D}}|f(z)|^{p} \mu(d z) \leq \sum_{\alpha \in T_{2}} \mu(\alpha)\left|f\left(z_{\alpha}\right)\right|^{p} .
$$

Condition (10) implies the discrete inequality

$$
\sum_{\alpha \in T_{2}} \mu(\alpha)\left|a_{\alpha}\right|^{p} \leq C \sum_{\alpha \in T_{2}} \lambda(\alpha)\left|a_{\alpha}\right|^{p}
$$

Now, there exists $\varepsilon>0$ such that, if $V_{\alpha}=\cup_{\beta \cap \alpha \neq \phi} \beta$, then $B\left(z_{\alpha}, \varepsilon(1-\right.$ $|z|)) \subseteq V_{\alpha}$. Thus, by (11), the mean value property, Jensen's inequality, and the regularity of $\rho$ we have

$$
\begin{aligned}
\int_{\mathbb{D}}|f(z)|^{p} \mu(d z) \\
\quad \leq C \sum_{\alpha \in T_{2}} \int_{\alpha} \rho(w) m(d w)\left|f\left(z_{\alpha}\right)\right|^{p} \\
\quad \leq C \sum_{\alpha \in T_{2}} \int_{\alpha} \rho(w) m(d w)\left|\frac{1}{\left(1-\left|z_{\alpha}\right|\right)^{2}} \int_{B\left(z_{\alpha}, \varepsilon\left(1-\left|z_{\alpha}\right|\right)\right)} f(z) m(d z)\right|^{p} \\
\quad \leq C \sum_{\alpha \in T_{2}} \int_{\alpha} \rho(w) m(d w) \frac{1}{\left(1-\left|z_{\alpha}\right|\right)^{2}} \int_{B\left(z_{\alpha}, \varepsilon\left(1-\left|z_{\alpha}\right|\right)\right)}|f(z)|^{p} m(d z) \\
\leq C \sum_{\alpha \in T_{2}} \int_{V_{\alpha}}|f(z)|^{p} \rho(z) m(d z) \\
\leq C \int_{\mathbb{D}}|f(z)|^{p} \rho(z) m(d z) .
\end{aligned}
$$

Thus, $\mu$ is Carleson for $A_{p}(\rho)$.

Suppose now that $\mu$ is Carleson for $A_{p}(\rho)$, i.e. that

$$
\text { Id }: A_{p}(\rho) \longrightarrow L^{p}(\mu)
$$


is bounded. By Theorem 6, this is equivalent to the boundedness of the adjoint, $\Theta=\mathrm{Id}^{*}$;

$$
\begin{gathered}
\Theta: L^{p^{\prime}}(\mu) \longrightarrow A_{p^{\prime}}\left(\rho^{1-p^{\prime}}\right), \\
\langle\Theta g, f\rangle_{A_{2}}=\langle g, f\rangle_{L^{2}(\mu)} .
\end{gathered}
$$

Explicitly,

$$
\Theta g(\xi)=\int_{\mathbb{D}} g(z) \overline{k_{\xi}(z)} \mu(d z),
$$

where $k_{\xi}(z)=(1-z \bar{\xi})^{-2}$ is the reproducing kernel for $A_{2}$; that is, $\left\langle f, k_{\xi}\right\rangle_{A_{2}}$ $=f(\xi)$, whenever $f \in A_{2}, \xi \in \mathbb{D}$. If we test the boundedness of $\Theta$ on functions of the form $g=\chi_{\alpha}, \alpha \in T_{2}$, we obtain

$$
\int_{\alpha}\left|\int_{\alpha} \frac{\mu(d z)}{(1-z \bar{\xi})^{2}}\right|^{p^{\prime}} \rho(\xi)^{1-p^{\prime}} m(d \xi) \leq C(\mu) \int_{\alpha} \mu(d z) .
$$

Now note that, for some $\delta>0$,

$$
\left|\int_{\alpha} \frac{\mu(d z)}{(1-z \bar{\xi})^{2}}\right| \geq \delta \frac{\mu(\alpha)}{m(\alpha)} .
$$

This follows from the fact that if $N$ is sufficiently large then there is an $\varepsilon>0$ such that if $z$ and $\xi$ are in the same $\Delta_{\alpha}$ then $\left|\arg (1-z \bar{\xi})^{-2}\right| \leq \pi / 2-\varepsilon$. From (13), and (12), one deduces (10).

With little more effort, one can characterize the measures for which

$$
\left(\int_{\mathbb{D}}|f(z)|^{q} \mu(d z)\right)^{1 / q} \leq C(\mu)\left(\int_{\mathbb{D}}|f(z)|^{p} \rho(z) m(d z)\right)^{1 / p}
$$

when $1<p<q<\infty$, for all $f \in A_{p}(\rho)$, where $\rho$ is an admissible weight. The condition on $\mu$ is, then,

$$
\mu(x)^{1 / q} \leq c \lambda(x)^{1 / p} .
$$

If $1<q \leq p<\infty$, Luecking [L2] showed that the condition is

$$
\sum_{x \in T_{2}} \mu(x)^{p /(p-q)} \lambda(x)^{-q /(p-q)} \leq C .
$$

Our duality argument does not seem to work in this case; however, see [A]. 
This proof of Luecking's theorem contains the main outline of the proof of Theorem 1. If we regard functions in $A_{p}(\rho)$ as being essentially constant on Whitney boxes and if we forget some of their structure (e.g., the mean value property) then condition (11) is a discrete analogue of the definition of Carleson measure for $A_{p}(\rho)$. In this case the associated discrete problem, characterizing $\mu$ 's and $\lambda$ 's so that (11) holds, is trivial. (11) is equivalent to (10). Together with the mean value property and Jensen's inequality, this fact is sufficient to show that (10) implies that $\mu$ is a Carleson measure for $A_{p}(\rho)$. To prove the converse, we test the boundedness of the adjoint operator on localized characteristic functions. For this to give us what we want, we must identify the dual space of $A_{p}(\rho)$ with respect to the duality pairing given by the inner product of $A_{2}$. That choice of pairing is dictated by its compatibility with the traditional $L^{p_{-}}$ $L^{p^{\prime}}$ duality for the function spaces on the tree $T$. The results of Bonami and Bekollé allow us to identify that dual space.

\section{Carleson measures on trees.}

In this section we prove Theorems 3 and 4 . We also consider some special cases in which it is especially easy to verify whether a measure $\nu$ on $T$ is Carleson for $(\mathcal{I}, p)$. We also give results comparing, when $p=q$, condition (6) with the simpler condition (7).

Let $T$ be a tree. We use the same name $T$ for the tree and for its set of vertices. We do not assume that $T$ is locally finite; a vertex of $T$ can be the endpoint of infinitely many edges. If $x, y \in T$, the geodesic between $x$ and $y,[x, y]$, is the set $\left\{x_{0}, \ldots, x_{n}\right\}$ where $x_{0}=x, x_{n}=y, x_{j-1}$ is adjacent to $x_{j}$ (i.e., $x_{j-1}$ and $x_{j}$ are endpoints of an edge), and the vertices in [x,y] are all distinct. We let $[x, x]=\{x\}$. If $x, y$ are as above, we let $d(x, y)=n$. Let $o \in T$ be a fixed root. We will say that $T$ is homogeneous of degree $k$ if $o$ is the endpoint of $k$ edges and every other vertex is the endpoint of $k+1$ edges. We say that $x \leq y, x, y \in T$, if $x \in[o, y]$. $\leq$ is a partial order on $T$. If $x \in T, x \neq o$, we denote by $x^{-} \in T$ the predecessor of $x: x^{-}<x$ and $d\left(x, x^{-}\right)=1$. We denote $d(x)=\sharp[o, x]=d(o, x)+1$. For $x \in T$, the Carleson square (or box) of vertex $x$ (or shadow of $x$ ) is $S(x)=\{y \in T: y \geq x\}$. For $x \in T$, we denote by $\delta_{x}$ the characteristic function of the singleton $x$, or, when convenient, the measure consisting of a point mass at $x$. Let $\mu$ be a positive measure on $T$. The inner product on $L^{2}(\mu)=L^{2}(T, \mu)$ is $\langle f, g\rangle_{L^{2}(\mu)}=\sum_{x \in T} f(x) \overline{g(x)} \mu(x)$. 
Proof of Theorem 3. Fix $p, q, 1<p \leq q<\infty$, and let $\mu$ be a Carleson measure for $(\mathcal{I}, \rho, p, q)$. Testing the boundedness of $\mathcal{I}$ on the function $\delta_{o}$ we deduce that $\mu$ must be bounded. $\mathcal{I}^{*}$, the formal adjoint of $\mathcal{I}$, is bounded from $L^{q^{\prime}}(\mu)$ to $L^{p^{\prime}}\left(\rho^{1-p^{\prime}}\right)$. Now, $\mathcal{I}^{*} f(x)=\sum_{y \in S(x)} f(y) \mu(y)$. Let $\chi_{E}$ be the characteristic function of the set $E$. If we test the boundedness of $\mathcal{I}^{*}$ on functions of the form $f=\chi_{S(r)}$, we obtain (6). This shows the only if part of the theorem.

Suppose now that $\mu$ is bounded and that (6) holds. We need to show that $\mathcal{I}: L^{p}(\rho) \longrightarrow L^{q}(\mu)$ is bounded, i.e., that

$$
\|\mathcal{I} g\|_{L^{q}(\mu)} \leq C\|g\|_{L^{p}(\rho)},
$$

for some $C>0$. It suffices to show that (16) holds for $g \geq 0$. In this case, $\mathcal{I} g$ is nondecreasing with respect to the order relation on $T$. Let

$$
\Omega_{k}=\left\{x: \mathcal{I} g(x)>2^{k}\right\}=\bigcup_{j} S\left(r_{j}^{k}\right)=\bigcup_{j} Q_{j}^{k}
$$

where $\left\{r_{j}^{k}: j=1, \ldots\right\} \subset T$ is the set of the minimal points in $\Omega_{k}$ with respect to the partial order on $T$ and $Q_{j}^{k}=S\left(r_{j}^{k}\right)$. We suppose that no $r_{j}^{k+1}$ coincides with an $r_{j}^{k}$ and leave for the reader the minor modifications for the general case.

Let $E_{j}^{k}=S\left(r_{j}^{k}\right) \cap\left(\Omega_{k+1} \backslash \Omega_{k+2}\right)$. Then, if $x \in E_{j}^{k}$,

$$
\mathcal{I}\left(\chi_{Q_{j}^{k}} g\right)(x)=\sum_{r_{j}^{k} \leq y \leq x} g(y)=\mathcal{I} g(x)-\mathcal{I} g\left(\left(r_{j}^{k}\right)^{-}\right)>2^{k+1}-2^{k}=2^{k}
$$

Here we let $\mathcal{I} g\left(\left(r_{j}^{k}\right)^{-}\right)=0$ if $\left(r_{j}^{k}\right)^{-}=o$. Thus,

$$
\begin{aligned}
2^{k} \mu\left(E_{j}^{k}\right) & =2^{k} \sum_{x \in E_{j}^{k}} \mu(x) \\
& \leq \sum_{x \in E_{j}^{k}} \mathcal{I}\left(\chi_{Q_{j}^{k}} g\right)(x) \mu(x) \\
& =\sum_{y \in Q_{j}^{k}} g(y) \sum_{x \in E_{j}^{k}, x \geq y} \mu(x) \\
& =\sum_{y \in Q_{j}^{k}} g(y) \mathcal{I}^{*} \chi_{E_{j}^{k}}(y) .
\end{aligned}
$$


Denoting the complement of $\Omega_{k+2}$ by $\Omega_{k+2}^{c}$ and noting that $\mathcal{I}^{*} \chi_{E_{j}^{k}}(y)=0$ when $y \in Q_{j}^{k} \cap \Omega_{k+2}$ we obtain

$$
2^{k} \mu\left(E_{j}^{k}\right) \leq \sum_{y \in Q_{j}^{k} \cap \Omega_{k+2}^{c}} g(y) \mathcal{I}^{*} \chi_{E_{j}^{k}}(y)
$$

Now,

$$
\begin{aligned}
\sum_{x \in T, \mathcal{I} g(x)>2}|\mathcal{I} g(x)|^{q} \mu(x) & \leq \sum_{k} \mu\left(x: 2^{k+1} \leq \mathcal{I} g(x)<2^{k+2}\right) 2^{q(k+2)} \\
& =C \sum_{k} \mu\left(\Omega_{k+1} \backslash \Omega_{k+2}\right) 2^{q k} \\
& \leq C \sum_{k, j} \mu\left(E_{j}^{k}\right) 2^{q k} \\
& =C\left(\sum_{(k, j) \in E}+\sum_{(k, j) \in F}\right) \mu\left(E_{j}^{k}\right) 2^{q k} \\
& =C\left(\sum_{1}+\sum_{2}\right) .
\end{aligned}
$$

Here,

$$
\begin{aligned}
& E=\left\{(k, j): \mu\left(E_{j}^{k}\right) \leq \beta \mu\left(Q_{j}^{k}\right)\right\}, \\
& F=\left\{(k, j): \mu\left(E_{j}^{k}\right)>\beta \mu\left(Q_{j}^{k}\right)\right\},
\end{aligned}
$$

and $\beta$ is a positive constant to be chosen later. In the inequalities below $C$ is a constant independent of $g$ and $\beta$. To estimate the first sum, note that, by definition of $\Omega_{k}$,

$$
\begin{aligned}
\sum_{1} & \leq \beta \sum_{(k, j)} \mu\left(Q_{j}^{k}\right) 2^{q k} \\
& =\beta \sum_{k} \mu\left(\Omega_{k}\right) 2^{q k} \\
& \leq C \beta \sum_{x \in T}|\mathcal{I} g(x)|^{q} \mu(x) \\
& =C \beta\|\mathcal{I} g\|_{L^{q}(\mu)}^{q} .
\end{aligned}
$$


On the other hand, we can estimate $\sum_{2}$ by

$$
\begin{aligned}
& \sum_{2}=\sum_{(k, j) \in F} \mu\left(E_{j}^{k}\right) 2^{q k} \\
&(\operatorname{by}(18)) \leq \sum_{(k, j) \in F} \mu\left(E_{j}^{k}\right)^{-1} \\
& \cdot\left|\mu\left(E_{j}^{k}\right)^{-1} \sum_{y \in Q_{j}^{k} \cap \Omega_{k+2}^{c}} g(y) \mathcal{I}^{*} \chi_{E_{j}^{k}}(y)\right|^{q}
\end{aligned}
$$$$
\text { (by definition of } F) \leq \beta^{-q} \sum_{(k, j)} \frac{\mu\left(E_{j}^{k}\right)}{\mu\left(Q_{j}^{k}\right)^{q}}\left|\sum_{y \in Q_{j}^{k} \cap \Omega_{k+2}^{c}} g(y) \mathcal{I}^{*} \chi_{E_{j}^{k}}(y)\right|^{q}
$$

(by Hölder's inequality) $\leq \beta^{-q} \sum_{(k, j)} \mu\left(Q_{j}^{k}\right)^{1-q}$

$$
\begin{gathered}
\cdot\left(\sum_{y \in Q_{j}^{k} \cap \Omega_{k+2}^{c}}\left|\mathcal{I}^{*} \chi_{E_{j}^{k}}(y)\right|^{p^{\prime}} \rho(y)^{1-p^{\prime}}\right)^{q / p^{\prime}} \\
\cdot\left(\sum_{y \in Q_{j}^{k} \cap \Omega_{k+2}^{c}}|g(y)|^{p} \rho(y)\right)^{q / p} \\
(\text { by }(6)) \leq C \beta^{-q} \sum_{(k, j)}\left(\sum_{y \in Q_{j}^{k} \cap \Omega_{k+2}^{c}}|g(y)|^{p} \rho(y)\right)^{q / p} \\
\text { (since } q \geq p) \leq C \beta^{-q}\left(\sum_{(k, j)} \sum_{y \in Q_{j}^{k} \cap \Omega_{k+2}^{c}}|g(y)|^{p} \rho(y)\right)^{q / p} \\
=C \beta^{-q}\left(\sum_{k} \sum_{\Omega_{k} \backslash \Omega_{k+2}}|g(y)|^{p} \rho(y)\right)^{q / p} \\
=C \beta^{-q}\left(\sum_{y \in T}|g(y)|^{p} \rho(y)\right)^{q / p} .
\end{gathered}
$$

From the estimates for $\Sigma_{1}$ and $\Sigma_{2}$, and a similar argument when $\mathcal{I} g(x) \leq 2$, we obtain that

$$
\sum_{x \in T}|\mathcal{I} g(x)|{ }^{q} \mu(x) \leq C \beta \sum_{x \in T}|\mathcal{I} g(x)|{ }^{q} \mu(x)+C \beta^{-q}\left(\sum_{y \in T}|g(y)|^{p} \rho(y)\right)^{q / p}
$$


By choosing $\beta$ small enough, we have that $\mathcal{I}$ is bounded.

The necessary condition in $3,(6)$, was obtained by testing $\mathcal{I}^{*}$ on the characteristic functions of the "natural" sets for the operator $\mathcal{I}^{*}$. We could have tested $\mathcal{I}$ itself on its "natural" functions, namely, $\varphi_{x}=\chi_{[0, x]} \rho^{1-p^{\prime}}$, $x \in T$. Those functions are natural for $\mathcal{I}$ in the sense that if $y \geq x$, we have the equality

$$
\left\|\varphi_{x}\right\|_{L^{p}(\rho)}^{p}=\mathcal{I} \varphi_{x}(y)
$$

Using those as test functions gives

Proposition 8. Let $1<p \leq q<\infty$ and let $\rho$ be a positive weight on $T$. If $\mu$ is Carleson for $(\mathcal{I}, \rho, p, q)$, then

$$
\left(\sum_{x \in S(z)} \mu(x)\right)^{1 / q} \leq C\left(\sum_{0}^{z} \rho(x)^{1-p^{\prime}}\right)^{-1 / p^{\prime}}
$$

with $C$ independent of $z \in T$. The constant is quantitative.

We will see in Section 8 that (19), which is sometimes called a single box condition, is, in fact, a discretization and generalization of the necessary and sufficient condition for a measure to be Carleson for the analytic Hardy space (or the Bergman space). It is interesting that for $p=q$ and a large class of weights (19) is not sufficient. We return to that after the proof of Theorem 4, a weak variant of which is next.

Theorem 9. Let $1<p<q<\infty$ and let $\rho$ be a weight on T. For a nonnegative function $\mu$ on $T$, the following are equivalent.

i) $\mathcal{I}$ maps $L^{p}(\rho)$ to weak $L^{q}(\mu)$, i.e.,

$$
\sup _{\lambda>0} \lambda \mu(x \in T: \mathcal{I} f(x) \geq \lambda)^{1 / q} \leq C(\mu)\left(\sum_{x \in T} f(x)^{p} \rho(x)\right)^{1 / p}
$$

whenever $f \geq 0$.

ii) (19) holds, for some $C>0$.

Proof that Theorem 9 implies Theorem 4. We proceed as in [Saw2, p. 341]. By Theorem 3, it suffices to show that the weak inequality (20) 
implies (6). For $r \in T$,

$$
\begin{aligned}
& \left(\sum_{x \in S(r)}\left(\sum_{y \geq x} \mu(y)\right)^{p^{\prime}} \rho(x)^{1-p^{\prime}}\right)^{1 / p^{\prime}} \\
& =\left(\sum_{x \in T}\left|\mathcal{I}^{*}\left(\chi_{S(r)} \mu\right)(x)\right|^{p^{\prime}} \rho(x)^{1-p^{\prime}}\right)^{1 / p^{\prime}} \\
& =\sup _{\|f\|_{L^{p}(\rho)} \leq 1} \sum_{x \in T} \mathcal{I}\left(\chi_{S(r)} \mu\right)(x) f(x) \\
& =\sup _{\|f\|_{L^{p}(\rho)} \leq 1} \sum_{y \in S(r)} \mathcal{I} f(y) \mu(y) \\
& =\sup _{\|f\|_{L^{p}(\rho)} \leq 1} \int_{0}^{\infty} \mu(S(r) \cap\{\mathcal{I} f>\lambda\}) d \lambda \\
& \text { by }(20) \leq C \int_{0}^{\infty} \min \left\{\mu(S(r)), \lambda^{-q}\right\} d \lambda \\
& =C \mu(S(r))^{1 / q^{\prime}} \text {, }
\end{aligned}
$$

which is (6).

Proof of Theorem 4. Let $\sigma=\rho^{1-p^{\prime}}$. Fix $r \in T$ and let $\lambda=\sigma([0, r])$. If

$$
f=\chi_{[0, r]} \sigma
$$

then $\mathcal{I} f(x)=\lambda$ for $x \in S(r)$ and the weak type inequality (20) yields

$$
\sigma([o, r]) \mu(S(r))^{1 / q} \leq C\left(\sum_{x \in T} f(x)^{p} \rho(x)\right)^{1 / p}=C \sigma([o, r])^{1 / p}
$$

since $\sigma^{p} \rho=\sigma$. Dividing both sides by $\sigma([o, r])^{1 / p}$ yields condition (7).

Conversely, fix $f \geq 0$ in $L^{p}(\rho)$. Let $r_{o}$ be a minimal point in the set $E(f, c \lambda / 2)=\{x \in T: \mathcal{I} f(x) \geq \lambda / 2\}$ and let $x \in S\left(r_{0}\right) \cap E(f, \lambda)$. Then

$$
\sum_{r_{0} \leq y \leq x} f(y)>\frac{\lambda}{2}
$$

Now, define $r_{0}<r_{1}<\cdots<x$ so that 
i) $\mu\left(S\left(r_{k+1}\right)\right)<\mu\left(S\left(r_{k}\right)\right) / 2$,

ii) $\mu(S(r)) \geq \mu\left(S\left(r_{k}\right)\right)$ for $r_{k}<r<r_{k+1}$.

i.e., $r_{k+1}$ is the first point along the path leading to $x$ for which its successor set has $\mu$-measure less than half that of the successor set of $r_{k}$. Of course, it is understood that the construction stops at, say, $r_{N}$, when

$$
\mu(S(x)) \geq \frac{1}{2} \mu\left(S\left(r_{k}\right)\right) .
$$

By (21),

$$
\frac{\lambda}{2}<\sum_{k=0}^{N} \sum_{r_{k} \leq y<r_{k+1}} f(y)
$$

where the sum stops at $x$ when $k=N$

$$
\begin{aligned}
& \leq \sum_{k=0}^{N}\left(\sum_{r_{k} \leq y<r_{k+1}} \sigma(y)\right)^{1 / p^{\prime}}\left(\sum_{r_{k} \leq y<r_{k+1}} f(y)^{p} \rho(y)\right)^{1 / p} \\
& \leq \sum_{k=0}^{N} \sigma\left(\left[o, t_{k+1}\right]\right)^{1 / p^{\prime}}\left(\sum_{y \in S\left(r_{k}\right)} f(y)^{p} \rho(y)\right)^{1 / p}
\end{aligned}
$$

where $t_{k+1}$ is the predecessor of $r_{k+1}$

$$
\begin{aligned}
& \text { by }(7) \leq C \sum_{k=0}^{N} \mu\left(S\left(t_{k+1}\right)\right)^{-1 / q}\left(\sum_{y \in S\left(r_{k}\right)} f(y)^{p} \mu(y)\right)^{1 / p} \\
&\text { by ii }) \leq C \sum_{k=0}^{N} \mu\left(S\left(r_{k}\right)\right)^{-1 / q}\left(\sum_{y \in S\left(r_{k}\right)} f(y)^{p} \mu(y)\right)^{1 / p} \\
& \leq C\left(\sum_{k=0}^{N} \mu\left(S\left(r_{k}\right)\right)^{1 / p-1 / q}\right) \sup _{r_{0} \leq t \leq x}\left(\frac{1}{\mu(S(t))} \sum_{y \in S(t)} f(y)^{p} \rho(y)\right)^{1 / p} \\
& \text { by i) } \leq C \mu\left(S\left(r_{0}\right)\right)^{1 / p-1 / q} \sup _{r_{0} \leq t \leq x}\left(\frac{1}{\mu(S(t))} \sum_{y \in S(t)} f(y)^{p} \rho(y)\right)^{1 / p} .
\end{aligned}
$$

Thus, to each point in $S\left(r_{0}\right) \cap E(f, \lambda)$ there corresponds a point $r_{x} \in\left[r_{0}, x\right]$ such that

$$
\lambda \leq C \mu\left(S\left(r_{0}\right)\right)^{1 / p-1 / q} \sup _{r_{0} \leq t \leq x}\left(\frac{1}{\mu(S(t))} \sum_{y \in S(t)} f(y)^{p} \rho(y)\right)^{1 / p}
$$


or, equivalently,

$$
\mu\left(S\left(r_{x}\right)\right) \leq C \lambda^{-p} \mu\left(S\left(r_{0}\right)\right)^{1-p / q} \sum_{y \in S\left(r_{x}\right)} f(y)^{p} \rho(y)
$$

Now, let $\left\{r_{i}\right\}_{i}$ be the minimal points in $S\left(r_{0}\right) \cap E(f, \lambda)$. Then, by (22),

$$
\begin{aligned}
\lambda^{q} \mu\left(S\left(r_{0}\right) \cap E(f, \lambda)\right) & =\lambda^{q} \sum_{i} \mu\left(S\left(r_{i}\right)\right) \\
& \leq C \lambda^{q-p} \mu\left(S\left(r_{0}\right)\right)^{1-p / q} \sum_{i} \sum_{y \in S\left(r_{i}\right)} f(y)^{p} \rho(y) \\
& \leq C \lambda^{q-p} \mu\left(S\left(r_{0}\right)\right)^{1-p / q} \sum_{y \in S\left(r_{0}\right)} f(y)^{p} \rho(y) .
\end{aligned}
$$

Summing over $r_{0} \in \mathcal{M}$, the set of the minimal points in $E(f, \lambda / 2)$,

$$
\begin{aligned}
\lambda^{q} \mu(E(f, \lambda)) & \leq C \lambda^{p-q} \sum_{r \in \mathcal{M}} \mu(S(r))^{1-p / q} \sum_{y \in S(r)} f(y)^{p} \rho(y) \\
& \leq C\left(\sum_{r \in \mathcal{M}} \mu(S(r))\right)^{1-p / q}\left(\sum_{r \in \mathcal{M}}\left(\sum_{y \in S(r)} f(y)^{p} \rho(y)\right)^{q / p}\right)^{p / q} \\
& \leq C\left(\lambda^{q} \mu\left(E\left(f, \frac{\lambda}{2}\right)\right)\right)^{1-p / q} \sum_{y \in T} f(y)^{p} \rho(y)
\end{aligned}
$$

For $t>0$, take the supremum over $0<\lambda<t$ in the last inequality, to get

$$
\sup _{0<\lambda<t} \lambda^{q} \mu(E(f, \lambda)) \leq C\left(\sup _{0<\lambda<t} \lambda^{q} \mu(E(f, \lambda))\right)^{1-p / q} \sum_{y \in T} f(y)^{p} \rho(y) .
$$

Standard arguments show that the first factor on the right hand side is finite under the hypothesis of the theorem. Algebraic rearrangement now gives the desired inequality. Now, divide both sides by the first factor the right hand side to obtain the desired inequality. Standard arguments show, in fact, that this factor is finite under the hypothesis of the theorem.

We now introduce a special class of Carleson measures we will use several times. We will assume that $p=q$ for the rest of this section. Let $x, y \in T$. The minimum between $x$ and $y, x \wedge y$, is the unique $t \in T$ such that $t \leq x, t \leq y$ and $t$ is maximum in $T$ with this property. A sequence 
$Z$ in $T$ is tree-like if $z \wedge w \in Z$ whenever $z \in Z$ and $w \in Z$. Let $\rho$ be a positive weight on $T$. For $x, y \in T$, let

$$
d_{\rho}(x, y)=\sum_{t \in[x, y]} \rho(t)^{1-p^{\prime}}
$$

and let

$$
d_{\rho}(x)=\sum_{o \leq t \leq x} \rho(t)^{1-p^{\prime}}
$$

The function $d_{\rho}$ has all properties of a distance except for the fact that $d_{\rho}(x, x) \neq 0$. Also note that when $\rho \equiv 1, d_{\rho}=d+1$ (not $d !$ ).

Many properties of the operator $\mathcal{I}$ can be expressed in terms of $d_{\rho}$. To each sequence $Z \subseteq T$ and to each $p \in(1, \infty)$ we associate the measure $\mu_{Z}$

$$
\mu_{Z}(x)=\sum_{z \in Z} d_{\rho}(z)^{1-p} \delta_{z}(x)
$$

Proposition 10. Let $Z$ be a tree-like sequence in $T$. Then, $\mu_{Z}$ is Carleson for $(\mathcal{I}, \rho, p)$ if and only if there exists $C>0$ so that

$$
\mu_{z}(S(z))=\sum_{w \in Z \cap S(z)} d_{\rho}(w)^{1-p} \leq C d_{\rho}(z)^{1-p}
$$

whenever $z \in Z$.

Proof. To see that condition (26) is necessary, it suffices to test the boundedness of $\mathcal{I}$ on functions of the form $\chi_{[o, z]} \rho^{1-p^{\prime}}, z \in Z$. On the other hand, condition (26) implies (6), and hence that $\mu_{Z}$ is a Carleson measure. To see this, observe that since $Z$ is tree-like to each $r \in T$ we can associate 
the smallest $\xi=\xi(r) \in Z$ such that $\xi \geq z$, and $S(r) \cap Z=S(\xi) \cap Z$. Hence

$$
\begin{aligned}
\sum_{x \in S(r)}\left|\sum_{y \in S(x)} \mu_{Z}(y)\right|^{p^{\prime}} \rho(x)^{1-p^{\prime}} & \\
& =\sum_{x \in S(\xi(r))}\left|\sum_{z \in S(\xi(x)) \cap Z} d_{\rho}(z)^{1-p}\right|^{p^{\prime}} \rho(x)^{1-p^{\prime}} \\
& =\sum_{w \in S(\xi(r)) \cap Z} \sum_{w^{-}<x \leq w} \rho(x)^{1-p^{\prime}}\left|\sum_{z \in S(w) \cap Z} d_{\rho}(z)^{1-p}\right|^{p^{\prime}} \\
& \leq C \sum_{w \in S(\xi(r)) \cap Z} d_{\rho}(w)^{(1-p) p^{\prime}} \sum_{w^{-}<x \leq w} \rho(x)^{1-p^{\prime}} \\
& \leq C^{2} \sum_{w \in S(\xi(r)) \cap Z} d_{\rho}(w)^{(1-p) p^{\prime}+1} \\
& =C^{2} \sum_{x \in S(r)} \mu_{Z}(x) .
\end{aligned}
$$

We now show that the single box condition (19) is generally not sufficient for a measure to be Carleson. This phenomenon was first noted by Adams [Ad] in the context of Sobolev spaces and by Stegenga [Ste] in his work on multipliers and Carleson measures for the Dirichlet space $B_{2}$. Our construction is reminiscent of Stegenga's argument.

For a radial weight $\rho$ on $T$ we define $\rho(n)$ by $\rho(n)=\rho(x)$ where $d(o, x)=n$.

Theorem 11. Let $T$ be a homogeneous tree of degree 2 with root o. Let $\rho$ be a radial weight on $T$. Let $1<p<\infty$. Assume that

i) $\rho(n) \leq C \rho(n+1)$ for some $C>0$ and all $n \geq 0$, and

ii) $\sum_{n=0}^{\infty} \rho^{1-p^{\prime}}(n)=\infty$.

Then there exists $\mu \geq 0$ on $T$ such that (19) holds, but $\mu$ is not a Carleson measure for $(\mathcal{I}, \rho, p)$.

Proof. We will construct a sequence $Z, Z \subseteq T$ and, as before, define a measure $\mu$ by letting $\mu(z)=d_{\rho}(z)^{1-p}$, if $z \in Z, \mu(x)=0$, if $x \notin Z$.

First we construct $W$, the sequence of the branching points of $Z$. Consider a tree-like sequence $W$ in $T$, homogeneous of degree 2 as a tree, 
having the same root as $T$ and having $2^{n}$ points $w_{1}^{n}, \ldots, w_{2^{n}}^{n}$ at the level $n$ in $W$, such that $d_{\rho}\left(w_{j}^{n}\right) \sim\left(n 2^{n}\right)^{1 /(p-1)}$. We will show later that there are such points.

Now we construct $Z$. For each $w \in W$ with $d_{\rho}(w) \sim\left(n 2^{n}\right)^{1 /(p-1)}$ we select $z=z(w)$ so that $z>w, d_{\rho}(z) \sim\left(n^{2} 2^{n}\right)^{1 /(p-1)}$, and whenever $\xi \in W, \xi>w$, one has that $z \wedge \xi=w$. The last condition can be restated as: if $x_{1}$ and $x_{2}$ are the points of $T$ immediately below $w$ and, say, $z \geq x_{1}$, then $S\left(x_{1}\right) \cap W=\varnothing$.

In this context, (19) and (6) read, respectively,

$$
\sum_{z \in Z, z \geq x} d_{\rho}(z)^{1-p} \leq C d_{\rho}(x)^{1-p}
$$

and

$$
\sum_{y \geq x}\left(\sum_{z \in Z, z \geq y} d_{\rho}(z)^{1-p}\right)^{p^{\prime}} \rho(y)^{1-p^{\prime}} \leq C \sum_{z \in Z, z \geq x} d_{\rho}(z)^{1-p} .
$$

It is easy to verify that (29) holds if and only if it holds for $x=w \in W$, a branching point. By our construction of $W$ and $Z,(29)$ can then be rephrased as: for all $h \geq 0$

$$
\sum_{k \geq h} 2^{k-h}\left(k^{2} 2^{k}\right)^{-1} \leq C\left(h 2^{h}\right)^{-1} .
$$

This is fine. On the other hand, (30) fails. To see this first note that, by (25), for $y \in\left[w_{j}^{k-1}, w_{l}^{k}\right]$ we have

$$
\rho(y)^{1-p^{\prime}} \backsim\left(\left(k 2^{k}\right)^{p^{\prime}-1}-\left((k-1) 2^{k-1}\right)^{p^{\prime}-1}\right) \backsim\left(k 2^{k}\right)^{p^{\prime}-1} .
$$

In this case (30) is equivalent to

$$
\sum_{k \geq h} 2^{k-h}\left(k 2^{k}\right)^{p^{\prime}-1}\left(\sum_{n \geq k} 2^{n-k}\left(n^{2} 2^{n}\right)^{-1}\right)^{p^{\prime}} \leq C \sum_{k \geq h} 2^{k-h}\left(k^{2} 2^{k}\right)^{-1}
$$

which simplifies as

$$
\sum_{k \geq h} 2^{-h}(k)^{p^{\prime}-1}\left(\sum_{n \geq k} n^{-2}\right)^{p^{\prime}} \leq C \sum_{k \geq h} 2^{-h}\left(k^{2}\right)^{-1},
$$

which simplifies as

$$
\sum_{k \geq h} k^{p^{\prime}-1}\left(k^{-1}\right)^{p^{\prime}} \leq C h^{-1}
$$


which fails.

It remains to verify that we can find $w_{j}^{n}$ 's and $z=z\left(w_{j}^{n}\right)^{\text {'s such that }}$

$$
d_{\rho}^{p-1}\left(w_{j}^{n}\right) \sim 2^{n} n, \quad d_{\rho}^{p-1}(z) \sim 2^{n} n^{2} .
$$

By assumption ii) in the hypothesis, there exists $N$ such that

$$
\sum_{k=0}^{N} \rho_{k}^{1-p^{\prime}} \geq\left(2^{n} n\right)^{p^{\prime}-1} \quad \text { and } \quad \sum_{k=0}^{N-1} \rho_{k}^{1-p^{\prime}}<\left(2^{n} n\right)^{p^{\prime}-1} .
$$

Hence, by i),

$$
\sum_{k=0}^{N} \rho^{1-p^{\prime}} \leq \sum_{k=0}^{N-1} \rho^{1-p^{\prime}}+C \rho_{N-1}^{1-p^{\prime}} \leq(1+C)\left(2^{n} n\right)^{p^{\prime}-1} .
$$

Thus,

$$
\left(\sum_{k=0}^{N} \rho^{1-p^{\prime}}\right)^{p-1} \sim 2^{n} n
$$

and we can choose $w$ such that $d(o, w)=N$. The same reasoning applies to the choice of $z(w)$.

Remark 1. With a slight modification of the proof, we can exhibit $\mu$ such that (19) holds and (6) fails if the following conditions are satisfied:

i) Each vertex in $T$ other than the root is the endpoint of at least three edges.

ii) $\rho\left(z^{-}\right) \leq C \rho(z)$, where $z^{-}$is the predecessor of $z$ in $T$.

iii) $\sum_{\xi=o}^{z} \rho(\xi)^{1-p^{\prime}} \longrightarrow \infty$ as $z \longrightarrow \partial T$, the boundary of $T$, along any geodesic.

Remark 2. If the tree reduces to the integers then (19) and (6) are equivalent.

We now look at two cases in which Proposition 10 is decisive. Let $Z$ be a tree-like sequence in a tree $T$. Assume, for simplicity, that $o \in Z$. We can think of $Z$ itself as a tree with root $o$. Suppose that $Z$ is homogeneous of degree $k$. Suppose that $\rho \equiv 1$, hence $d_{\rho}=d+1$. Suppose that $d_{Z}$, the 
edge-counting distance in the tree $Z$, satisfies $d(z) \backsim d_{Z}(o, z)^{m}$ for some $m>1$. An inspection of (26) shows that $\mu_{Z}$ is a Carleson measure for $(\mathcal{I}, p)$ if and only if

$$
p>1+\frac{\log k}{\log m} .
$$

More generally, if $d(z)=\phi\left(d_{Z}(o, z)\right)$, where $\phi$ is an increasing, positive function, then $\mu_{Z}$ is Carleson for $(\mathcal{I}, p)$ if and only if for all $j$

$$
\sum_{n \geq j} k^{n} \phi(n)^{1-p} \leq C k^{j} \phi(j)^{1-p}
$$

where $C>0$ is independent of $j$.

Now suppose that $Z=T$ is a homogeneous tree of degree $k$ and that the weight $\rho$ is defined by $\rho(z)=k^{a d(o, z)}$ for some $a \in \mathbb{R}$. This weight corresponds to a weight $\rho$ on $\mathbb{D}$, which is given by $\rho(z)=\left(1-|z|^{2}\right)^{-a}$. Then, $\mu_{Z}$ is Carleson for $(\mathcal{I}, \rho, p)$ if and only if $a>0$. Observe that, in this case, hypothesis ii) of Theorem 11 is not satisfied.

\section{Carleson measures for the analytic Besov spaces.}

\subsection{Carleson measures.}

In this section, we prove an equivalent formulation of Theorem 1, Theorem 12 below, and Theorem 2. Then, in Theorem 15, we give the characterization of the multipliers for the spaces $B_{p}(\rho)$.

For this section, recall $T_{2}$, the tree introduced in Section 1.

Theorem 12. Let $1<p \leq q<\infty$ and let $\rho$ be a p-admissible weight. A positive Borel measure $\mu$ on $\mathbb{D}$ is a Carleson measure for $\left(B_{p}(\rho), q\right)$ if and only if

$$
\left(\sum_{\beta \geq \alpha}\left(\sum_{\gamma \geq \beta} \mu(\gamma)\right)^{p^{\prime}} \rho(\beta)^{1-p^{\prime}}\right)^{q^{\prime} / p^{\prime}} \leq C \sum_{\beta \geq \alpha} \mu(\beta)
$$

for some constant $C=C(\mu)$ independent of $\alpha \in T_{2}$.

Proof. We prove first that condition (31) is sufficient, under the less restrictive hypothesis that $\rho$ is a regular weight. Let $f$ be holomorphic in 
D. Select $z_{\alpha}, w_{\alpha} \in \bar{\alpha}$ so that $\sup _{z \in \alpha}|f(z)|=\left|f\left(z_{\alpha}\right)\right|$ and $\sup _{w \in \alpha}\left|f^{\prime}(w)\right|=$ $\left|f^{\prime}\left(w_{\alpha}\right)\right|$. Set $\Phi(\alpha)=\left|f\left(z_{\alpha}\right)\right|$. Then

$$
\int_{\mathbb{D}}|f(z)|^{q} \mu(d z)=\sum_{\alpha \in T_{2}} \int_{\alpha}|f(z)|^{q} \mu(d z) \leq \sum_{\alpha \in T_{2}} \mu(\alpha)|\Phi(\alpha)|^{q} .
$$

Now set $\phi(o)=\Phi(o)$ and, for other points of $T_{2}$, set $\phi(\alpha)=\Phi(\alpha)-\Phi\left(\alpha^{-}\right)$, $\rho(\alpha)=\rho\left(z_{\alpha}\right)$. We have $\Phi(\alpha)=\mathcal{I} \phi(\alpha)$ and, by (31), Theorem 3, and the fact that $\rho$ is regular

$$
\left(\int_{\mathbb{D}}|f(z)|^{q} \mu(d z)\right)^{p / q} \leq C \sum_{\alpha \in T_{2}} \rho(\alpha)|\phi(\alpha)|^{p} .
$$

For $\alpha \neq o$,

$$
\begin{aligned}
|\phi(\alpha)| & =\left|\Phi(\alpha)-\Phi\left(\alpha^{-}\right)\right| \\
& =\left|\int_{z\left(\alpha^{-}\right)}^{z(\alpha)} f^{\prime}(w) d w\right| \\
& \leq C\left(\left|f^{\prime}\left(w_{\alpha}\right)\right|+\left|f^{\prime}\left(w_{\alpha^{-}}\right)\right|\right) \operatorname{diam}(\alpha),
\end{aligned}
$$

while for $\alpha=o$ we have

$$
\phi(o)=\left|f\left(z_{o}\right)\right| \leq|f(0)|+\left|\int_{0}^{z(o)} f^{\prime}(w) d w\right| \leq\left|f^{\prime}\left(w_{o}\right)\right| \operatorname{diam}(o)+|f(o)| .
$$

Summing over $\alpha \in T_{2}$, and noting that $\sharp\left\{\alpha \in T_{2}: \beta=\alpha^{-}\right\}=2$, for $\beta \neq o$ we obtain

$$
\left(\int_{\mathbb{D}}|f(z)|^{q} \mu(d z)\right)^{p / q} \leq C \sum_{\alpha \in T_{2}} \rho(\alpha)\left|f^{\prime}\left(w_{\alpha}\right)\right|^{p}+C \rho(o)|f(0)|^{p} .
$$

By the mean value property,

$$
f^{\prime}\left(w_{\alpha}\right)=\frac{1}{m\left(B_{\alpha}\right)} \int_{B_{\alpha}} f^{\prime}(z) m(d z)
$$

where $B_{\alpha}=B\left(w_{\alpha}, r_{\alpha}\right), r_{\alpha}=(1-|\alpha|) / 3$. Note $\sharp\left\{\beta \in T_{2}: B_{\alpha} \cap \beta \neq \varnothing\right\} \leq 4$ and hence $m\left(B_{\alpha}\right)$ is comparable with $m(\alpha)$. Thus, by Jensen's inequality,

$$
\left|f^{\prime}\left(w_{\alpha}\right)\right|^{p} \leq C \frac{1}{m(\alpha)} \int_{\cup_{\beta \cap B_{\alpha} \neq \varnothing \beta}}\left|f^{\prime}(z)\right|^{p} m(d z)
$$


and hence

$$
\begin{aligned}
\left(\int_{\mathbb{D}}|f(z)|^{q} \mu(d z)\right)^{p / q} & \leq C \sum_{\alpha \in T_{2}} \frac{\rho(\alpha)}{m(\alpha)} \int_{\alpha}\left|f^{\prime}(z)\right|^{p} m(d z) \operatorname{diam}(\alpha)^{p}+|f(0)|^{p} \\
& \leq C \sum_{\alpha \in T_{2}} \int_{\alpha}\left|\left(1-|z|^{2}\right) f^{\prime}(z)\right|^{p} \rho(z) m_{h}(d z)+C|f(0)|^{p} \\
& =C\|f\|_{B_{p}(\rho)}^{p}
\end{aligned}
$$

as wished.

Before we prove the necessity part, we need some preparation. Let $F, G$ be holomorphic functions in $\mathbb{D}$,

$$
F(z)=\sum_{0}^{\infty} a_{n} z^{n}, \quad G(z)=\sum_{0}^{\infty} b_{n} z_{n} .
$$

Define

$$
\langle F, G\rangle_{\mathcal{D}^{*}}=\sum_{1}^{\infty} n a_{n} \overline{b_{n}}=\int_{\mathbb{D}} F^{\prime}(z) \overline{G^{\prime}(z)} m(d z)
$$

and

$$
\langle F, G\rangle_{\mathcal{D}}=a_{0} \overline{b_{0}}+\sum_{1}^{\infty} n a_{n} \overline{b_{n}}=F(0) \overline{G(0)}+\langle F, G\rangle_{\mathcal{D}^{*}}
$$

Lemma 13. Let $\rho$ be a weight satisfying (1). Then $B_{p^{\prime}}\left(\rho^{1-p^{\prime}}\right)$ is the dual of $B_{p}(\rho)$ under the pairing $\langle\cdot, \cdot\rangle_{\mathcal{D}}$, i.e., each functional $\Lambda$ on $B_{p}(\rho)$ can be represented as

$$
\Lambda f=\langle f, g\rangle_{\mathcal{D}}, \quad f \in B_{p}(\rho),
$$

for a unique $g \in B_{p^{\prime}}\left(\rho^{1-p^{\prime}}\right)$.

Lemma 13 is a direct consequence of Theorem 6 and the definition of the $B_{p}$.

A simple manipulation of Fourier series shows that the reproducing kernel of $\mathcal{D}$ with respect to the product $\langle\cdot, \cdot\rangle_{\mathcal{D}}$ is

$$
\phi_{z}(w)=1+\log \frac{1}{1-w \bar{z}}
$$

i.e., if $f \in \mathcal{D}$, then

$$
f(z)=\left\langle f, \phi_{z}\right\rangle_{\mathcal{D}}=\int_{\mathbb{D}} f^{\prime}(w) \overline{\left(1+\log \frac{1}{1-\bar{z} w}\right)^{\prime}} m(d w)+f(0) .
$$


Lemma 14. Let $\rho$ be an admissible weight, $1<p<\infty$. Then $\phi_{z}$ is a reproducing kernel for $B_{p^{\prime}}\left(\rho^{1-p^{\prime}}\right)$; i.e., if $G \in B_{p^{\prime}}\left(\rho^{1-p^{\prime}}\right)$ then

$$
G(z)=\left\langle G, \phi_{z}\right\rangle_{\mathcal{D}}
$$

In particular, point evaluation is bounded on $B_{p^{\prime}}\left(\rho^{1-p^{\prime}}\right)$.

Observe that $(1)$ is symmetric in $(\rho, p)$ and $\left(\rho^{1-p^{\prime}}, p^{\prime}\right)$ and hence the same conclusion holds for $B_{p}(\rho)$.

Proof. For fixed $z$,

$$
\begin{aligned}
\left\|\phi_{z}\right\|_{B_{p}(\rho)}^{p} & =1+\int_{\mathbb{D}}\left|\frac{\left(1-|w|^{2}\right) \bar{z}}{1-\bar{z} w}\right|^{p} \rho(w) m_{h}(d w) \\
& \sim 1+\int_{\mathbb{D}}\left(1-|w|^{2}\right)^{p-2} \rho(w) m(d w)
\end{aligned}
$$

with a constant that depends on $z$. Since (1) holds, the integral is finite and hence $\phi_{z} \in B_{p}(\rho)$. (32) follows from a Fourier series argument.

Now let $\mu$ be a positive bounded measure on $\mathbb{D}$ and define

$$
\langle F, G\rangle_{\mu}=\langle F, G\rangle_{L^{2}(\mu)}=\int_{\mathbb{D}} F(z) \overline{G(z)} \mu(d z) .
$$

$\mu$ is Carleson for $\left(B_{p}(\rho), p, q\right)$ if and only if

$$
\text { Id }: B_{p} \longrightarrow L^{q}(\mu)
$$

is bounded. In turn, this is equivalent to the boundedness, with the same norm, of its adjoint $\Theta=\mathrm{Id}^{*}$,

$$
\Theta: L^{q^{\prime}}(\mu) \longrightarrow\left(B_{p}(\rho)\right)^{*} \equiv B_{p^{\prime}}\left(\rho^{1-p^{\prime}}\right) .
$$

Here we have used the duality pairings $\langle\cdot, \cdot\rangle_{\mathcal{D}}$ and $\langle\cdot, \cdot\rangle_{\mu}$ and Lemma 13.

By Lemma 14,

$$
\begin{aligned}
\Theta G(z) & =\left\langle\Theta G, \phi_{z}\right\rangle_{\mathcal{D}} \\
& =\left\langle G, \phi_{z}\right\rangle_{L^{2}(\mu)} \\
& =\int_{\mathbb{D}}\left(1+\log \frac{1}{1-z \bar{w}}\right) G(w) \mu(d w) .
\end{aligned}
$$


Now let $\mu$ be Carleson for $\left(B_{p}(\rho), q\right)$. Fix $\alpha \in T_{2} \backslash\{o\}$ and define

$$
g(w)=\chi_{S(\alpha)}(w) \bar{w}^{-1}
$$

Since $\mu$ is Carleson and $S(\alpha)$ has distance at least $1 / 2$ from the origin of D,

$$
\begin{aligned}
\left(\sum_{\beta \geq \alpha} \mu(\beta)\right)^{p^{\prime} / q^{\prime}} \\
\quad=\mu(S(\alpha))^{p^{\prime} / q^{\prime}} \\
\quad \geq C\|g\|_{L^{q^{\prime}(\mu)}}^{p^{\prime} q^{\prime}} \\
\quad \geq C(\mu)\|\Theta g\|_{B_{p^{\prime}}\left(\rho^{1-p^{\prime}}\right)}^{p^{\prime}} \\
\quad \geq C \int_{\mathbb{D}}\left(1-|z|^{2}\right)^{p^{\prime}} \rho(z)^{1-p^{\prime}}\left|\int_{\mathbb{D}} g(w) \frac{\bar{w}}{1-z \bar{w}} \mu(d w)\right|^{p^{\prime}} m_{h}(d z) \\
\quad \geq C \int_{\mathbb{D}} \rho(z)^{1-p^{\prime}}\left|\int_{S(\alpha)} \frac{1-|z|^{2}}{1-z \bar{w}} \mu(d w)\right|^{p^{\prime}} m_{h}(d z)
\end{aligned}
$$

Now

$$
\operatorname{Re} \frac{1-|z|^{2}}{1-z \bar{w}}=\frac{1-|z|^{2}}{|1-z \bar{w}|^{2}} \operatorname{Re}(1-\bar{z} w) \geq 0
$$

and, if $z \in \widetilde{S}(\alpha), w \in S(z) \cap S(\alpha)$ and $\theta=\arg (\bar{z} w)$, then

$$
\begin{aligned}
\operatorname{Re} \frac{1-|z|^{2}}{1-z \bar{w}} & =\frac{\left(1-|z|^{2}\right)(1-|z||w| \cos \theta)}{(1-|z||w|)^{2}+2|z||w|(1-\cos \theta)} \\
& \geq C \frac{\left(1-|z|^{2}\right)^{2}}{\max \left\{\left(1-|z|^{2}\right)^{2}, \theta^{2}\right\}} \\
& \geq \delta \\
& >0
\end{aligned}
$$

with $\delta$ independent of $\alpha$.

Inserting this estimate in the previous chain of inequalities, we obtain

$$
\left(\sum_{\beta \geq \alpha} \mu(\beta)\right)^{p^{\prime} / q^{\prime}} \geq C \int_{\widetilde{S}(\alpha)} \rho(z)^{1-p^{\prime}}\left(\int_{S(z) \cap S(\alpha)} \mu(d w)\right)^{p^{\prime}} m_{h}(d z)
$$


We can split the integral over $\widetilde{S}(\alpha)$ as

$$
\begin{aligned}
\int_{\widetilde{S}(\alpha)} \mu(S(z) \cap S(\alpha))^{p^{\prime}} \rho(z)^{1-p^{\prime}} m_{h}(d z) & \\
= & \sum_{\beta \geq \alpha} \int_{\beta} \mu(S(z) \cap S(\alpha))^{p^{\prime}} \rho(z)^{1-p^{\prime}} m_{h}(d z) \\
& \quad+\int_{\widetilde{S}(\alpha) \backslash S(\alpha)} \mu(S(z) \cap S(\alpha))^{p^{\prime}} \rho(z)^{1-p^{\prime}} m_{h}(d z) .
\end{aligned}
$$

Since the Whitney boxes $\beta$ and $\widetilde{S}(\alpha) \backslash S(\alpha)$ have comparable hyperbolic area, and $\rho$ is essentially constant in each box, the latter is

$$
\begin{aligned}
& \geq C \sum_{\beta \geq \alpha} \rho(\beta)^{1-p^{\prime}}\left(\sum_{\gamma>\beta} \mu(\gamma)\right)^{p^{\prime}}+C \rho(\alpha)^{1-p^{\prime}}\left(\sum_{\gamma \geq \alpha} \mu(\gamma)\right)^{p^{\prime}} \\
& \geq \sum_{\beta \geq \alpha} \rho(\beta)^{1-p^{\prime}}\left(\sum_{\gamma \geq \beta} \mu(\gamma)\right)^{p^{\prime}} .
\end{aligned}
$$

Hence,

$$
\left(\sum_{\beta \geq \alpha} \mu(\beta)\right)^{p^{\prime} / q^{\prime}} \geq C \sum_{\beta \geq \alpha} \rho(\beta)^{1-p^{\prime}}\left(\sum_{\gamma \geq \beta} \mu(\gamma)\right)^{p^{\prime}}
$$

as wished.

When $\alpha=o$, a similar argument works.

Proof of Theorem 2. By Theorems 1, 3 and 4, when $p<q$, (2) is equivalent to (7), which is equivalent to (4).

\subsection{Multipliers.}

Let $\rho$ be a positive weight on $\mathbb{D}$ and $1<p<\infty$. Given $\varphi$, a function holomorphic in $\mathbb{D}$, we say that $\varphi$ is a multiplier of $B_{p}(\rho)$ if

$$
f \longmapsto \varphi f
$$

is a bounded operator from $B_{p}(\rho)$ into itself. We write $\|\varphi\|=\|\varphi\|_{M}$ for the norm of the multiplication operator, and $\varphi \in M=M\left(B_{p}(\rho)\right)$.

The characterization of the multipliers of $B_{p}$ is given in [Wang] and $[\mathrm{Wu}]$, following the argument given in [Ste] for $p=2$. We show that their results extend to allow admissible weights. 
Theorem 15. Let $1<p<\infty$, let $\rho$ be an admissible weight. Let $\varphi$ be a holomorphic function on $\mathbb{D}$ and define

$$
\mu_{\varphi}(d z)=(1-|z|)^{p-2}\left|\varphi^{\prime}(z)\right|^{p} \rho(z) m(d z)=(\delta \varphi)^{p} \rho m_{h}(d z) .
$$

Then, $\varphi \in M\left(B_{p}(\rho)\right)$ if and only if $\varphi$ is bounded and $\mu_{\varphi}$ is a Carleson measure for $B_{p}(\rho)$.

Proof. Let $\lambda_{z}, z \in \mathbb{D}$, be the point evaluation in $\mathbb{D}$. Since $\rho$ is admissible, Lemma $14 \lambda_{z}$ is bounded on $B_{p}(\rho)$. Suppose $\varphi \in M\left(B_{p}(\rho)\right)$. Let $\left(B_{p}(\rho)\right)^{*}$ be the dual space of $B_{p}(\rho)$ and $\langle\cdot, \cdot\rangle$ be the duality product. Then, if $f$ is holomorphic in $\mathbb{D}$,

$$
\begin{aligned}
|\varphi(z) f(z)| & =\left|\left\langle\varphi f, \lambda_{z}\right\rangle\right| \\
& \leq\|\varphi f\|_{B_{p}(\rho)}\left\|\lambda_{z}\right\|_{\left(B_{p}(\rho)\right)^{*}} \\
& \leq\|\varphi\|_{M}\|f\|_{B_{p}(\rho)}\left\|\lambda_{z}\right\|_{\left(B_{p}(\rho)\right)^{*}} .
\end{aligned}
$$

Taking the supremum of $|f(z)|$ over those $f$ for which $\|f\|_{B_{p}(\rho)}=1$,

$$
|\varphi(z)|\left\|\lambda_{z}\right\|_{\left(B_{p}(\rho)\right)^{*}} \leq\|\varphi\|_{M}\left\|\lambda_{z}\right\|_{\left(B_{p}(\rho)\right)^{*}}
$$

and hence $\varphi$ is bounded.

If $\varphi$ is a multiplier of $B_{p}(\rho)$ then

$$
\left(\int_{\mathbb{D}}\left|(\varphi f)^{\prime}(z)\right|^{p}\left(1-|z|^{2}\right)^{p-2} \rho(z) m(d z)\right)^{1 / p} \leq C\|f\|_{B_{p}(\rho)} .
$$

Because $\varphi$ is bounded this implies

$$
\left(\int_{\mathbb{D}}|f(z)|^{p}\left|\varphi^{\prime}(z)\right|^{p}\left(1-|z|^{2}\right)^{p-2} \rho(z) m(d z)\right)^{1 / p} \leq C\|f\|_{B_{p}(\rho)}
$$

i.e., $\mu_{\varphi}$ is Carleson for $B_{p}(\rho)$.

Conversely, if $\varphi$ is bounded, then

$$
|\varphi(0) f(0)| \leq\|\varphi\|_{L^{\infty}}|f(0)|
$$

Also, if $\mu_{\varphi}$ is Carleson for $B_{p}(\rho)$, then

$$
\left(\int_{\mathbb{D}}|f(z)|^{p}\left|\varphi^{\prime}(z)\right|^{p}\left(1-|z|^{2}\right)^{p-2} \rho(z) m(d z)\right)^{1 / p} \leq C\|f\|_{B_{p}(\rho)}^{p}
$$


and, since $\varphi$ is bounded,

$$
\begin{aligned}
\left(\int_{\mathbb{D}}\left|\varphi(z) f^{\prime}(z)\right|^{p}\left(1-|z|^{2}\right)^{p-2} \rho(z) m(d z)\right)^{1 / p} & \\
& \leq C\left(\int_{\mathbb{D}}\left|f^{\prime}(z)\right|^{p}\left(1-|z|^{2}\right)^{p-2} \rho(z) m(d z)\right)^{1 / p} \\
& \leq C\|f\|_{B_{p}(\rho)}^{p} .
\end{aligned}
$$

Combining these we find

$$
\left(\int_{\mathbb{D}}\left|(\varphi f)^{\prime}(z)\right|^{p}\left(1-|z|^{2}\right)^{p-2} \rho(z) m(d z)\right)^{1 / p} \leq C\|f\|_{B_{p}(\rho)}^{p}
$$

i.e., $\mu_{\phi}$ is Carleson for $B_{p}(\rho)$.

\section{Various characterizations of Carleson measures.}

In this section we compare various noncapacitary conditions on weights and give some examples. In Proposition 16 we show that condition (3) in Theorem 1 and the discrete condition (31) in Theorem 12 are equivalent, hence completing the proof of Theorem 1 . Next, when $p=q$, we look at the relation between the more complicated condition (2) and the single box condition. In Proposition 18, we show that a single box condition is implied by the more complicated (3), but, by Theorem 19, is generally not equivalent to it. In the third part we compare our conditions with those of $[\mathrm{KS}]$ which we recall as Theorem 21. We show in Proposition 22 that, for purely measure theoretic reasons, the condition (3) on the measure $\mu$ implies the corresponding condition in [KS]. However, in fact, the two conditions are equivalent and that implies the equivalence of a range of conditions for a measure to be a Carleson measure. That is in Corollary 23. The issue of constructing families of examples of admissible weights is addressed in Lemma 17 and Proposition 20 and in the final subsection. Those examples may be familiar to experts but we include them in the hope they will help illuminate the general landscape and the various comparisons being discussed. In Proposition 24, we give a conformally invariant characterization of the Carleson measures for $B_{p}(\rho)$. 


\subsection{Equivalence of the discrete and continuous con- ditions.}

To complete the proof of Theorem 1 we need to show that the condition (3) in Theorem 1 and condition (31) in Theorem 12, which a priori might depend on the Whitney decomposition of the disc, are in fact equivalent.

Proposition 16. Let $\rho$ be a regular weight. Then (3) and (31) are equivalent. Namely, for a measure $\mu$ on $\mathbb{D}$ the following are equivalent:

1)

$$
\left(\int_{\widetilde{S}(a)} \rho(z)^{-p^{\prime} / p}(\mu(S(z) \cap S(a)))^{p^{\prime}} m_{h}(d z)\right)^{q^{\prime} / p^{\prime}} \leq C_{1} \mu(S(a)),
$$

for some constant $C_{1}$ independent of $a \in \mathbb{D}$.

2)

$$
\left(\sum_{\beta \geq \alpha}\left(\sum_{\gamma \geq \beta} \mu(\gamma)\right)^{p^{\prime}} \rho(\beta)^{1-p^{\prime}}\right)^{q^{\prime} / p^{\prime}} \leq C \sum_{\beta \geq \alpha} \mu(\beta)
$$

for some constant $C$ independent of $\alpha \in T_{2}$.

Proof. Suppose that (35) holds for $\mu$. We specialize the inequality to $I=I(\alpha)$. Then

$$
\mu(S(I))=\sum_{\beta \geq \alpha} \mu(\beta)
$$

while, since $\rho$ is regular,

$$
\begin{aligned}
&\left(\int_{S(I)} \rho(z)^{-p^{\prime} / p}(\mu(S(z)\right.\left.\cap S(a)))^{p^{\prime}} m_{h}(d z)\right)^{q^{\prime} / p^{\prime}} \\
& \sim\left(\sum_{\beta \geq \alpha} \rho(\beta)^{1-p^{\prime}} \int_{\beta} \mu(S(z) \cap S(I))^{p^{\prime}} m_{h}(d z)\right)^{q^{\prime} / p^{\prime}} \\
& \geq c\left(\sum_{\beta \geq \alpha} \rho(\beta)^{1-p^{\prime}}\left(\sum_{\gamma \geq \beta} \mu(\gamma)\right)^{p^{\prime}}\right)^{q^{\prime} / p^{\prime}} .
\end{aligned}
$$


The same reasoning shows that

$$
\begin{aligned}
\left(\int_{\widetilde{S}(I) \backslash S(I)} \rho(z)^{-p^{\prime} / p}(\mu(S(z) \cap S(a)))^{p^{\prime}}\right. & \left.m_{h}(d z)\right)^{q^{\prime} / p^{\prime}} \\
& \geq\left(\rho(\alpha)^{1-p^{\prime}}\left(\sum_{\gamma \geq \alpha} \mu(\gamma)\right)^{p^{\prime}}\right)^{q^{\prime} / p^{\prime}} .
\end{aligned}
$$

As a consequence, (36) holds.

Suppose, conversely, that (36) holds. Hence by Theorem 3, (36) holds for $\mu_{I}=\left.\mu\right|_{S(I)}$, with a smaller constant. Thus, if $\alpha$ is such that $\widetilde{S}(I) \subseteq$ $S(\alpha)$,

$$
\begin{aligned}
C \mu(S(I)) & \geq\left(\sum_{\beta \geq \alpha} \rho(\beta)^{1-p^{\prime}}\left(\sum_{\gamma \geq \beta} \mu_{I}(\gamma)\right)^{p^{\prime}}\right)^{q^{\prime} / p^{\prime}} \\
& =\left(\sum_{\beta \geq \alpha} \rho(\beta)^{1-p^{\prime}} \mu_{I}(S(\beta))^{p^{\prime}}\right)^{q^{\prime} / p^{\prime}}
\end{aligned}
$$

Now, if $z \in \beta$, it is easy to see that

$$
\mu_{I}(S(z)) \leq \mu_{I}(S(\beta))+\mu_{I}\left(S\left(\beta_{1}\right)\right)+\mu_{I}\left(S\left(\beta_{2}\right)\right)
$$

where $\beta_{1}$ and $\beta_{2}$ are boxes at the same level of $\beta$ and adjacent to it. We can then estimate the previous sum by

$$
\begin{aligned}
& \geq c\left(\sum_{\beta \geq \alpha} \rho(\beta)^{1-p^{\prime}} \int_{\beta} \mu_{I}(S(z))^{p^{\prime}} m_{h}(d z)\right)^{q^{\prime} / p^{\prime}} \\
& \geq c\left(\int_{S(I)} \frac{\mu_{I}(S(z))^{p^{\prime}}}{\rho(z)^{p^{\prime}-1}\left(1-|z|^{2}\right)^{2}}\right)^{q^{\prime} / p^{\prime}} .
\end{aligned}
$$

Condition (35) now follows from this chain of inequalities and from the fact that, again by (36),

$$
C \mu(S(\alpha)) \geq \int_{\widetilde{S}(I) \backslash S(I)} \frac{\mu_{I}(S(z))^{p^{\prime}}}{\rho(z)^{p^{\prime}-1}\left(1-|z|^{2}\right)^{2}} .
$$




\subsection{Relation with the Carleson condition.}

Let $\rho_{\alpha}(z)=\left(1-|z|^{2}\right)^{\alpha}$ and let $\mathcal{D}_{\alpha}$ be the associated scale of Dirichlet spaces; $\mathcal{D}_{\alpha}=B_{2}\left(\rho_{\alpha}(z)\right)$. In 1980 Stegenga [Ste] characterized the Carleson measures for $\mathcal{D}_{\alpha}, \alpha \geq 0$. His result includes Carleson's classical theorem for $H^{2} \equiv \mathcal{D}_{1}$ and a characterization of Carleson measures for the Bergman space $A_{2} \equiv \mathcal{D}_{2}$. When $\alpha \geq 1$, a necessary and sufficient condition for $\mu$ to be Carleson for $\mathcal{D}_{\alpha}$ is the single box condition

$$
\mu(S(z)) \rho_{\alpha}^{-1}(z) \leq C
$$

For the $\rho_{\alpha},(37)$ is equivalent to

$$
\mu(S(z)) \int_{[0, z]} \rho_{\alpha}^{-1}(w) \frac{|d w|}{1-|w|^{2}} \leq C
$$

and it is this latter version which we want for later comparison. Stegenga also found that in the range $\alpha<1,(38)$ is not sufficient anymore, although still necessary. He gave a necessary and sufficient condition for $\mu$ to be a Carleson measure for $\alpha$ in the range $[0,1)$ in terms of a capacitary estimate. His result was later generalized by I. Verbitsky, [Ve], J. Wang [Wang] and $\mathrm{Wu}[\mathrm{Wu}]$ to $B_{p}, 1<p<\infty$.

We now show that this failure of the simple condition (38) is rather general. Of course our analysis only covers admissible weights. In particular, in the scale of spaces $\mathcal{D}_{\alpha}$, our analysis allows $-1<\alpha<1$.

Lemma 17. $\rho_{\alpha}$ is an admissible weight if and only if $-1<\alpha<1$.

Proof. We only have to verify the $\mathcal{B}_{2}$ condition. For $\rho_{\alpha}$, that reduces to

$$
\left(R \int_{0}^{R} t^{\alpha} d t\right)\left(R \int_{0}^{R} t^{-\alpha} d t\right) \leq C R^{4}
$$

which is satisfied for $|\alpha|<1$.

Remark. When $\alpha<0, \mathcal{D}_{\alpha}$ is contained in the space $C(\cdot)$ of the functions that are continuous on the closure of the unit disc. Hence, a measure is Carleson if and only if it is bounded. In particular, condition (35) is equivalent to the boundedness of $\mu$, which is equivalent to (38).

We now note that an analog of (38) holds in general. We also note in passing that this proposition indicates that, in the continuous case as well 
as the discrete case, underlying the analysis on $B_{p}(\rho)$, there is a distance which depends on $\rho$.

Proposition 18. Let $\rho$ be a regular weight. If $\mu$ satisfies (35), then

$$
\mu(S(z))^{1 / p}\left(\int_{[0, z]} \rho(w)^{1-p^{\prime}} \frac{|d w|}{1-|w|^{2}}\right)^{1 / p^{\prime}} \leq C
$$

for some constant $C$, independent of $z \in \mathbb{D}$.

Proof. By Proposition 16 and Theorem 3, (35) implies that $\mu$ is a Carleson measure for $(\mathcal{I}, \rho, p)$. The result then follows from Proposition 8 and the regularity of $\rho$.

Theorem 19. Let $1<p<\infty$, let $\rho$ be an admissible weight on $\mathbb{D}$, and suppose that

$$
\int_{0}^{e^{i \theta}} \rho(w)^{1-p^{\prime}} \frac{|d w|}{1-|w|^{2}}=\infty
$$

for all $e^{i \theta} \in \partial \mathbb{D}$.

Then, there exists a positive measure $\mu$ on $\mathbb{D}$ such that (39) holds, but $\mu$ is not a Carleson measure for $B_{p}(\rho)$.

Proof. By Theorem 11 there is a measure, $\mu$, defined on the tree $T_{2}$ such that (39) holds, but $\mu$ is not a Carleson measure for $(\mathcal{I}, \rho, p)$. By Theorem 3 and Theorem $12, \mu$, thought of as a measure on $\mathbb{D}$, is also not a Carleson measure for $B_{p}(\rho)$.

\subsection{Comparison with the condition of Kerman and Sawyer.}

In 1984 Kerman and Sawyer [KS] extended some of Stegenga's results; giving a necessary and sufficient condition for a measure to be a Carleson measure that does not make use of capacities. They worked with $p=2$ and considered Dirichlet weights on $\mathbb{D} ; \rho$ of the form $\rho(z)=\varphi(1-|z|)$ with $0 \leq \varphi \leq 1$, and $\varphi$ nondecreasing and concave. For instance, an easy calculation shows that $\rho_{\alpha}$ is a Dirichlet weight if and only if $0 \leq \alpha \leq 1$. All Dirichlet weights are regular but they are not all admissible; recall for instance that $\rho_{\alpha}$ is an admissible weight if and only if $-1<\alpha<1$. The intersection of the two classes is described by the following: 
Proposition 20. Let $\rho$ be a Dirichlet weight, $\rho(z)=\varphi(1-|z|)$. Then $\rho$ is admissible if and only if there are constants $C>0, \varepsilon \in(0,2)$, such that

$$
\varphi\left(2^{k} x\right) \leq C(2-\varepsilon)^{k} \varphi(x)
$$

whenever $k \geq 1$ is an integer and $2^{k} x \leq 1$.

Proof. Since $\varphi$ is increasing and concave,

$$
\varphi(2 x) \sim \varphi(x)
$$

hence,

$$
R \varphi(R) \sim \int_{0}^{R} \varphi(t) d t
$$

Condition $\mathcal{B}_{2}$ then reduces to

$$
\frac{1}{R} \int_{0}^{R} \varphi(t)^{-1} d t \leq C \varphi(R)^{-1}
$$

On the other hand,

$$
\int_{0}^{R} \varphi(t)^{-1} d t=\sum_{n=0}^{\infty} \int_{2^{-n-1} R}^{2^{-n} R} \varphi(t)^{-1} d t \sim \sum_{n=0}^{\infty} 2^{-n} R \varphi\left(2^{-n} R\right)^{-1}
$$

Thus, (41) is equivalent to

$$
\sum_{n=m}^{\infty} a_{n} \leq C a_{m}
$$

where

$$
a_{n}=2^{-n} \varphi\left(2^{-n}\right) .
$$

It is easy to prove that (42) holds if and only if

$$
a_{m+k} \leq \frac{C}{(1+\delta)^{k}} a_{m}
$$

for some $\delta, C>0$. 
It is clear that (43) implies (42). Conversely, if (42) holds, then

$$
\begin{aligned}
C a_{m} & \geq \sum_{n \geq m} a_{n}=a_{m}+\sum_{n \geq m+1} a_{n} \\
& \geq\left(1+\frac{1}{C}\right) \sum_{n \geq m+1} a_{n} \\
& \geq \cdots \\
& \geq\left(1+\frac{1}{C}\right)^{k} \sum_{n \geq m+k} a_{n}
\end{aligned}
$$

and (43) follows with $\delta=1 / C$. Now, (43) is a restatement of (40).

Theorem 21. (Kerman, Sawyer $[\mathrm{KS}]$ ). Let $\rho$ be a Dirichlet weight. Then, $\mu$ is Carleson for $\mathcal{D}_{\rho}=B_{2}(\rho)$ if and only if for all arcs $I$ on the boundary of the unit disk

$$
\int_{I} \sup _{\theta \in J \subseteq I} \frac{\mu(S(J))^{2}}{\rho(|J|)|J|} d \theta \leq C \mu(S(I))
$$

the supremum being taken over all closed arcs $J \subseteq I$.

Thus by Carleson's theorem for $H^{2}$ (i.e., $\rho=\rho_{1}$ ) we have that, for $\alpha=1,(38)$ is equivalent to (44). On the other hand, for $\rho=\rho_{\alpha}, 0 \leq \alpha<1$, (38) is strictly weaker than (44). In fact, we saw in Theorem 19 that for this range of $\alpha,(38)$ is strictly weaker than (31) which, by Theorem 12 and Lemma 17, is equivalent to (44). In some sense, then, the condition of Kerman and Sawyer is intermediate between the simple (38) and the more complicated (31) in Theorem 12.

Let $p=2$ and let $\rho$ be a Dirichlet weight. Because (44) and (35) are, respectively, necessary and sufficient for a measure $\mu$ to be Carleson for $\mathcal{D}(\rho)$, the latter must imply the former. In fact, as we show in the next proposition, (35) implies (44) is actually a consequence of the inclusion $l^{1} \subset l^{\infty}$ and holds for each interval $I$ separately. By similar reasoning, (44) must imply (35). However we don't know of a simple direct proof of that implication. (Since this manuscript was completed some further progress has been made, see $[\mathrm{AR}]$.

Proposition 22. Let $\rho$ be a regular weight on $\mathbb{D}$. If a positive measure $\mu$ satisfies (35), with $p=q$, then

$$
\int \sup _{I} \sup _{\theta \in I(z)}\left(\frac{\mu(S(z) \cap S(I))}{\rho(z)^{1 / p}|I(z)|^{1 / p^{\prime}}}\right)^{p^{\prime}} d \theta \leq C \mu(S(I)) .
$$


In particular, if $\rho$ is a regular weight and $p=2$, then (35) implies (44).

Proof. If $I$ is an arc on the boundary of $\mathbb{D}$,

$$
\int_{\widetilde{S}(I)} \frac{\mu(S(z) \cap S(I))^{p^{\prime}}}{\rho(z)^{p^{\prime}-1} m(S(z))} m(d z) \sim \int_{I} B(\theta) d \theta,
$$

where

$$
B(\theta)=\int_{1-2|I|}^{1} \frac{\mu\left(S\left(r e^{i \theta}\right) \cap S(I)\right)^{p^{\prime}}}{\rho\left(r e^{i \theta}\right)^{p^{\prime}-1}(1-r)^{2}} d r .
$$

Now,

$$
\begin{aligned}
B(\theta) & =\sum_{n=0}^{\infty} \int_{|I| / 2^{n}}^{|I| / 2^{n-1}} \frac{\mu\left(S\left((1-t) e^{i \theta}\right) \cap S(I)\right)^{p^{\prime}}}{\rho\left((1-t) e^{i \theta}\right) p^{p^{\prime}-1} t^{2}} d t \\
& \geq \sum_{n=0}^{\infty} \frac{\mu\left(S\left(\left(1-2^{-n}|I|\right) e^{i \theta}\right) \cap S(I)\right)^{p^{\prime}}}{\rho\left(\left(1-2^{-n}|I|\right) e^{i \theta}\right)^{p^{\prime}-1}\left(2^{-n}|I|\right)^{2}} 2^{-n}|I| \\
& \geq \sup _{n \geq 0} \frac{\mu\left(S\left(\left(1-2^{-n}|I|\right) e^{i \theta}\right) \cap S(I)\right)^{p^{\prime}}}{\rho\left(\left(1-2^{-n}|I|\right) e^{i \theta}\right)^{p^{\prime}-1} 2^{-n}|I|} \\
& \sim \sup _{\theta \in I(z)} \sup _{z \in S(I)} \frac{\mu(S(z) \cap S(I))^{p^{\prime}}}{\rho(z)^{p^{\prime}-1}|I(z)|} .
\end{aligned}
$$

Integrating over $I$ with respect to $\theta$ and using (35), we obtain (45).

In the other direction, suppose that $p=2$ and $\rho$ is an admissible weight and a Dirichlet weight. Then, if we know (45) holds uniformly for all intervals $I$, then the reverse implication holds. This shows that, in some sense, the inclusion $l^{1} \subset l^{\infty}$ can be reversed in that case. More specifically set

$$
a_{n}(I, \theta)=\frac{\mu\left(S\left(\left(1-2^{-n}|I|\right) e^{i \theta}\right) \cap S(I)\right)^{2}}{\rho\left(\left(1-2^{-n}|I|\right) e^{i \theta}\right) 2^{-n}|I|} .
$$

For $1 \leq q \leq \infty$ we are interested in the conditions,

There is a $C_{q}=C_{q}(\rho, \mu)$ so that for all arcs $I \subset \partial \mathbb{D}$,

$$
\begin{aligned}
\frac{1}{|I|} \int_{I}\left(\sum_{n=0}^{\infty} a_{n}(I, \theta)^{q}\right)^{1 / q} d \theta & \leq C_{q} \frac{1}{|I|} \int_{I} a_{0}(I, \theta) d \theta \\
& \backsim C_{q} \frac{1}{|I|} \mu(S(I)) .
\end{aligned}
$$


with the usual interpretation when $q=\infty$.

Corollary 23. Suppose $p=2, \rho$ is an admissible weight, and $\mu$ is a measure on $\mathbb{D}$. The following are equivalent:

1) $\mu$ is a Carleson measure for $B_{2}(\rho)$.

2) $\mathcal{C}_{q}$ holds for $q=1$.

3) $\mathcal{C}_{q}$ holds for some $q, 1 \leq q \leq \infty$.

4) $\mathcal{C}_{q}$ holds for all $q, 1 \leq q \leq \infty$.

5) $\mathcal{C}_{q}$ holds for all $q, 1 \leq q \leq \infty$ and the constants $C_{q}$ can be chosen to be independent of $q$.

6) $\mathcal{C}_{q}$ holds for $q=\infty$.

Proof. By Theorem 3, 1) implies that $\mu$ satisfies $(45)$. We saw in the proof of the previous proposition that (45) implies 2). By logic and Hölder's inequality, 2) implies each of 3), 4), and 5) and each of them implies 6). By the theorem of Kerman and Sawyer, 6) implies 1).

This corollary is reminiscent of the fact that the space BMO can be defined using $q^{\text {th }}$ power means for any $q, 1 \leq q<\infty$. The corollary also suggests questions such as the following: If these conditions hold then does it also hold, say, that there is a constant $C$ so that for all $\operatorname{arcs} I \subset \partial \mathbb{D}$

$$
\frac{1}{|I|} \int_{I} \sum_{n=0}^{\infty} a_{n}(I, \theta) d \theta \leq C \frac{1}{|I|} \int_{I}\left(\sum_{n=0}^{\infty} a_{n}(I, \theta)^{2}\right)^{1 / 2} d \theta
$$

\subsection{The condition of Evans, Harris, and Pick.}

In [EHP] Evans, Harris, and Pick show that the weighted Hardy inequality holds on a tree, i.e. (5) holds, if and only if $\mu$ satisfies a capacity condition thus giving a result which can be seen as a discrete analog of Luecking's theorem. It follows of course that their condition must be equivalent to our condition (6). However we don't know of a way to show that equivalence directly. 


\subsection{Examples.}

In these examples we work with Dirichlet weights and with the notation of Proposition 20. We will call $\varphi$ admissible if $\rho$ is.

1) Let $\varphi(t)=t^{\alpha} \log ^{\beta}(2 / t), \alpha, \beta \in$. Then $\varphi$ is admissible if and only if $\alpha<1$.

2) More generally, if $\varphi(t)=t / \varepsilon(t)$, where $\varepsilon(t) \longrightarrow 0$ as $t \longrightarrow 0$, then, $\varphi$ is admissible if and only if for some $C, \delta>0$

$$
\varepsilon\left(2^{-k} s\right) \leq C \varepsilon(s)(1+\delta)^{-k}
$$

In particular, if, for each $\alpha>0, \varphi(t)=O\left(t^{1-\alpha}\right)$, then $\varphi$ is not admissible.

3) If, for some $\varepsilon \in(0,1)$,

$$
\lim _{t \rightarrow 0} \frac{t \varphi^{\prime}(t)}{\varphi(t)} \leq 1-\varepsilon
$$

then $\varphi$ is admissible.

4) There exists a Dirichlet weight $\varphi$ such that, for $0<t \leq 1$,

$$
t^{1 / 2} \leq \varphi(t) \leq t^{1 / 4}
$$

but $\varphi$ is not admissible. This shows that it is not enough to impose a rough growth condition on $\varphi$ in order to make it admissible.

Here is an outline of the construction of such a $\varphi$. Let $u_{0}=1$, consider the straight line tangent to the graph of $s=t^{1 / 2}$ at $u_{0}$ and let $w_{1}<$ $u_{0}$ be the abscissa of the point where the line crosses the graph of $s=$ $t^{1 / 4}$. Consider the other tangent to $s=t^{1 / 2}$ passing through $\left(w_{1}, w_{1}^{1 / 4}\right)$, let $\left(u_{1}, u_{1}^{1 / 2}\right)$ be the point where it touches $s=t^{1 / 2}, u_{1}<w_{1}$, and let $\left(w_{2}, w_{2}^{1 / 4}\right)$ be the second point where this second line cuts the graph of $s=$ $t^{1 / 4}$. If we iterate this procedure, we find a sequence of points $\left(u_{0}, u_{0}^{1 / 2}\right)$, $\left(w_{1}, w_{1}^{1 / 4}\right),\left(u_{1}, u_{1}^{1 / 2}\right), \ldots$ Let $\varphi$ be the function on $[0,1]$, whose graph is the union of the segments joining these points. $\varphi$ is clearly a Dirichlet weight, taking on values between $t^{1 / 2}$ and $t^{1 / 4}$.

One easily verifies that $w_{n}=4^{2 / 3} u_{n}^{2 / 3}(1+o(1))$, as $n \longrightarrow \infty$. If $\varphi$ is admissible then by Proposition 20, with $x=u_{n}$ and $2^{k} x=w_{n}$, there would exist $\alpha \in(0,1)$ and $C>0$ such that

$$
\log \varphi\left(w_{n}\right)-\log \varphi\left(u_{n}\right) \leq C+\alpha\left(\log w_{n}-\log u_{n}\right) .
$$


(Actually, to do that we need $k$ to be an integer, we leave that technical adjustment to the reader.) This would imply that

$$
\log u_{n}\left(\frac{1}{6}-\frac{1}{2}\right) \leq C+\alpha \log u_{n}\left(\frac{2}{3}-1\right)
$$

which is absurd.

\subsection{A conformally invariant definition of Carleson measures.}

The inequality that defines Carleson measures, (2), is equivalent to a conformally invariant inequality, the equivalence being non quantitative.

Proposition 24. Given a Borel, probability measure $\mu$ on $\mathbb{D}$, a positive weight $\rho$ on $\mathbb{D}$ and $q \geq p$, the following are equivalent:

i) $\mu$ is a Carleson measure for $\left(B_{p}(\rho), q\right)$, i.e.,

$$
\left(\int_{\mathbb{D}}|f|^{q} d \mu\right)^{p / q} \leq C_{1}\left(\|f\|_{B_{p}(\rho)}^{* p}+|f(0)|^{p}\right) .
$$

ii) There exists $C_{2}>0$ such that

$$
\left(\int_{\mathbb{D}}|f-\mu(f)|^{q} d \mu\right)^{p / q} \leq C_{2}\|f\|_{B_{p}(\rho)}^{* p} .
$$

Here, $\mu(f)$ denotes the integral of $f$ with respect to $\mu$. Inequality (48) is conformally invariant in the following sense. If $\varphi$ is a conformal transformation of $\mathbb{D}$ and $\mu$ is a measure on $\mathbb{D}$, let $\varphi_{*} \mu(E)=\mu\left(\varphi^{-1}(E)\right)$ whenever $E \subseteq \mathbb{D}$. Similarly, if $\rho$ is a positive weight on $\mathbb{D}$, let $\varphi_{*} \rho(z)=$ $\rho\left(\varphi^{-1}(z)\right) . \varphi_{*}$ is, in both cases, the push-forward operator. Then, if (48) holds for $\mu$ and $\rho$, it holds, with the same constant, for $\varphi_{*} \mu$ and $\varphi_{*} \rho$. Also, observe that, if $\mu$ is a Dirac delta, then (48) holds with $C_{2}=0$, while we have $C_{1}>0$ in (47).

Proof. (47) implies (48). Applying (47) twice, we have

$$
\begin{aligned}
\left(\int_{\mathbb{D}}|f-\mu(f)|^{q} d \mu\right)^{p / q} & \leq C_{1}\left(\|f\|_{B_{p}(\rho)}^{* p}+|f(0)-\mu(f)|^{p}\right) \\
& \leq C_{1}\left(C_{1}+1\right)\|f\|_{B_{p}(\rho)}^{* p} .
\end{aligned}
$$


(48) implies (47). Observe first that (48) implies that

$$
\left(\int_{\mathbb{D}}|f|^{p} d \mu\right)^{p / q} \leq C_{3}\left(\|f\|_{B_{p}(\rho)}^{* p}+\mu(|f|)^{p}\right)
$$

with $C_{3} \leq 2^{p-1}\left(C_{2}+1\right)$. Suppose now that, by contradiction, (49) holds and (47) does not. Then, there exists a sequence $\left\{f_{n}\right\}$ of functions holomorphic in $\mathbb{D}$ such that

a) $\left|f_{n}(0)\right|^{p}+\left\|f_{n}\right\|_{B_{p}(\rho)}^{* p}=1$,

b) $\mu\left(\left|f_{n}\right|^{p}\right) \nearrow \infty$,

c) $\mu\left(\left|f_{n}\right|^{p}\right) \leq C \mu\left(\left|f_{n}\right|\right)^{p}$ for some $C>0$.

By normal families, we can assume that $f_{n}$ converges uniformly on compacts to some $g$ such that $\|g\|_{B_{p}(\rho)} \leq 1$. Let $M_{n}=\mu\left(\left|f_{n}\right|\right)$ and choose $r_{n} \nearrow 1$ such that

$$
\int_{|z| \leq r_{n}}\left|f_{n}\right| d \mu \geq \frac{M_{n}}{2}
$$

For fixed $s \in(0,1)$,

$$
\frac{f_{n} \chi_{\left\{|z| \leq r_{n}\right\}}}{M_{n}} \longrightarrow 0
$$

as $n \longrightarrow \infty$, uniformly on $\{|z| \leq s\}$. Thus, for $n \geq n(s)$,

$$
\int_{|z| \leq s}\left|f_{n}\right| d \mu \leq \frac{1}{4} M_{n}
$$

Hence,

$$
\int_{s \leq|z| \leq r_{n}}\left|f_{n}\right| d \mu \geq \frac{1}{4} M_{n}
$$

so that

$$
\frac{M_{n}^{p}}{4^{p}} \leq\left(\int_{s \leq|z| \leq r_{n}}\left|f_{n}\right| d \mu\right)^{p} \leq \int_{|z|<1}\left|f_{n}\right|^{p} d \mu\left(\int_{|z| \geq s} d \mu\right)^{p / p^{\prime}}
$$

i.e.,

$$
\mu\left(\left|f_{n}\right|^{p}\right) \geq \frac{\mu\left(\left|f_{n}\right|\right)^{p}}{4^{p} \mu(|z| \geq s)^{p / p^{\prime}}} .
$$

But this inequality contradicts c), since $\mu(|z| \geq s) \longrightarrow 0$ as $s \longrightarrow 1$. 


\section{Applications to discrete analysis.}

Let $T$ be a tree, $1<p<\infty$, and let $\rho$ be a positive weight on $T$. We define the Besov spaces $B_{p}(\rho)$ with respect to the root o on $T$ as the set of the functions $\Phi: T \longrightarrow \mathbb{C}$ such that

$$
\Phi=\mathcal{I} \phi
$$

for some $\phi \in L^{p}(\rho)$. We also write $\Phi^{\prime}=\phi$. The Besov $p$-norm of $\Phi \in B_{p}(\rho)$ is

$$
\|\Phi\|_{B_{p}(\rho)}=\|\phi\|_{L^{p}(\rho)} .
$$

This space is a discrete model for the space of holomorphic functions which we also denoted $B_{p}(\rho)$. In Theorem 25 and Theorem 26 we will discuss multipliers and interpolating sequences for $B_{p}(\rho)$.

We say that a positive measure $\nu$ on $T$ is Carleson for $B_{p}(\rho)$, or, simply, $p$-Carleson, if it is Carleson for $(\mathcal{I}, \rho, p)$. As in the classical case, $\nu$ is $p$-Carleson if and only if $B_{p}(\rho) \subseteq L^{p}(\nu)$ or, equivalently, if

$$
\|\Phi\|_{L^{p}(\nu)} \leq C(\nu)\|\Phi\|_{B_{p}(\rho)}
$$

A function $\Psi: T \longrightarrow \mathbb{C}$ is a multiplier of $B_{p}(\rho)$ if the map

$$
\Phi \longmapsto \Psi \Phi
$$

is bounded from $B_{p}(\rho)$ to itself.

Theorem 25. $\Psi$ is a multiplier of $B_{p}(\rho)$ if and only if $\Psi \in L^{\infty}(T)$ and the measure

$$
\nu=\left|\Psi^{\prime}\right|^{p} \rho
$$

is a Carleson measure for $B_{p}(\rho)$.

When we pass from spaces of holomorphic functions on $\mathbb{D}$ to this discrete model, $m_{h}(d z)$ is modeled by counting measure on $T$. Thus this result is a complete analog of Theorem 15 in Section 4.

Proof. The derivative' satisfies the product rule

$$
(\Psi \Phi)^{\prime}(x)=\Psi^{\prime}(x) \Phi(x)+\Psi(x) \Phi^{\prime}\left(x^{-}\right) .
$$


Suppose that $\Psi=\mathcal{I} \psi$ is a multiplier for $B_{p}(\rho)$, fix $z \in T$ and consider $\Phi_{z}=\mathcal{I} \delta_{z}$. By (51), $\left(\Psi \Phi_{z}\right)^{\prime}(z)=\Psi(z)$, hence

$$
\rho(z)=\left\|\Phi_{z}\right\|_{B_{p}(\rho)} \geq \frac{1}{C}\left\|\left(\Psi \Phi_{z}\right)^{\prime}\right\|_{L^{p}(\rho)} \geq \frac{1}{C} \rho(z)|\Psi(z)|
$$

and hence $\Psi \in L^{\infty}(T)$. Thus, for general $\Phi=\mathcal{I} \phi \in B_{p}(\rho)$,

$$
\begin{aligned}
C\|\Phi\|_{B_{p}(\rho)}^{p} & \geq\|\Psi \Phi\|_{B_{p}(\rho)}^{p} \\
& \geq \sum_{x \in T}|\psi(x)|^{p}|\Phi(x)|^{p} \rho(x)-\|\Psi\|_{L^{\infty}(T)}^{p} \sum_{x \in T}|\phi(x)|^{p} \rho(x),
\end{aligned}
$$

i.e., $\nu(x)=|\psi(x)|^{p} \rho(x)$ is a $p$-Carleson measure.

Conversely, suppose $\nu(x)$ is a $p$-Carleson measure. Then, by (51),

$$
\begin{aligned}
\|\Psi \Phi\|_{B_{p}(\rho)}^{p} & \leq C \sum_{x \in T}|\psi(x)|^{p}|\Phi(x)|^{p} \rho(x)+\|\Psi\|_{L^{\infty}(T)}^{p}\|\phi\|_{L^{p}(\rho)}^{p} \\
& \leq C\|\phi\|_{L^{p}(\rho)}^{p}=C\|\Phi\|_{B_{p}(\rho)}^{p} .
\end{aligned}
$$

Recall (24); for $x, y \in T$, we have $d_{\rho}(x, y)=\sum_{t \in[x, y]} \rho(t)^{1-p^{\prime}}$ and $d_{\rho}(x)=d_{\rho}(o, x)$.

Consider a sequence $Z \subset T$. Let $\nu=\nu_{Z}$ be the measure $\nu=$ $\sum_{z \in Z} d_{\rho}(z)^{1-p} \delta_{z} . Z$ is interpolating for $B_{p}(\rho)$ if and only if

$$
\text { Id }: B_{p}(\rho) \longrightarrow L^{p}\left(\nu_{Z}\right)
$$

is bounded and onto. This is equivalent to requiring the same of $\mathcal{I}$ : $L^{p}(\rho) \longrightarrow L^{p}\left(\nu_{Z}\right)$ or, with a different normalization, the same of the operator $A_{p}$ from $L^{p}(\rho)$ to $L^{p}(Z)$ given by

$$
A_{p} \phi(z)=d_{\rho}(z)^{-1 / p^{\prime}} \mathcal{I} \phi(z) .
$$

We use this formulation in the proof of the next theorem.

Theorem 26. For $Z \subset T$ and $1<p<\infty$, the following are equivalent:

1) $Z$ is interpolating for $B_{p}(\rho)$.

2) $\nu_{Z}$ is p-Carleson and there is a constant $A>0$ such that, for all $z, w \in Z, z \neq w$,

$$
A\left(d_{\rho}(z, w)-\rho(z \wedge w)^{1-p^{\prime}}\right) \geq d_{\rho}(z) .
$$


We want to rewrite this last condition in a way that emphasizes the analogy with later results. Suppose $\rho \equiv 1$. In this case (53) is equivalent to the requirement that there is are constants $A, B>0$ such that, for all $z, w \in Z, z \neq w$

$$
d(0, z) \leq A d(z, w)+B .
$$

The equivalence is straightforward after recalling that, for this $\rho, d_{\rho}(z, w)$ $=d(z, w)+1$; and noting that if $z \neq w$ then $d(z, w) \geq 1$. Thus, for $p=2$, this theorem is a perfect analog of Theorem BMS.

It is also interesting to note that, here as later, our solution to this interpolation problem is linear.

Proof. First we show that 1) implies 2). $\nu_{Z}$ must be Carleson by definition. By the open mapping theorem there is $C>0$ such that, for any $z \in Z$, there exists $\psi_{z} \in L^{p}(\rho)$ such that

$$
\mathcal{I} \psi_{z}(z)=d_{\rho}(z)^{1 / p^{\prime}} \delta_{z}, \quad\left\|\psi_{z}\right\|_{L^{p}(\rho)} \leq C
$$

Hence,

$$
\begin{aligned}
d_{\rho}(z)^{1 / p^{\prime}} & =\left|\mathcal{I} \psi_{z}(z)-\mathcal{I} \psi_{z}(w)\right| \\
& \leq\left|\sum_{z \wedge w<x \leq z} \psi_{z}(x)-\sum_{z \wedge w<x \leq w} \psi_{z}(x)\right| \\
& \leq\left\|\psi_{z}\right\|_{L^{p}(\rho)}\left(\sum_{x \in[z, w], x \neq z \wedge w} \rho(x)^{1-p^{\prime}}\right)^{1 / p^{\prime}} \\
& \leq C\left(d_{\rho}(z, w)-\rho(z \wedge w)^{1-p^{\prime}}\right)^{1 / p^{\prime}} .
\end{aligned}
$$

We now show that (2) implies (1). The proof consists in constructing, for $z \in Z$, a solution $\phi_{z}$ to

$$
\mathcal{I} \phi_{z}=\delta_{z}, \quad \text { for } z \in Z
$$

with

$$
\left\|\phi_{z}\right\|_{L^{p}(\rho)} \leq C d_{\rho}(z)^{-1 / p^{\prime}}
$$

and in such a way that each $x \in T$ belongs to the support of $\phi_{z}$ for at most two values of $z$. This allows us to construct solutions $\phi \in L^{p}(\rho)$ to

$$
\mathcal{I} \phi(z)=g(z), \quad z \in Z, g \in L^{p}\left(\nu_{Z}\right)
$$


In fact, let $\phi=\sum_{z \in Z} g(z) \phi_{z}$. Then (58) holds and, using the information about the overlap of the supports of the $\phi_{z}$ 's, we have

$$
\begin{aligned}
\|\phi\|_{L^{p}(\rho)}^{p} & =\sum_{x \in T} \rho(x)\left|\sum_{z \in Z} g(z) \phi_{z}(x)\right|^{p} \\
& =\sum_{x \in T} \rho(x)\left|\sum_{z: \phi_{z}(x) \neq 0} g(z) \phi_{z}(x)\right|^{p} \\
& \leq 2^{p} \sum_{z \in Z} g(z) \sum_{x: \phi_{z}(x) \neq 0} \phi_{z}(x)^{p} \rho(x) \\
& \leq C\|g\|_{L^{p}\left(\nu_{Z}\right)}^{p} .
\end{aligned}
$$

Thus the map $g \longmapsto \phi$ gives an explicit right inverse to $A_{p}=d(\cdot)^{-1 / p^{\prime}} \mathcal{I}$.

In order to show we can find the required functions $\phi_{z}$ such that (56) and (57) hold we endow $Z$ with a suitable tree-like structure. Assume, for

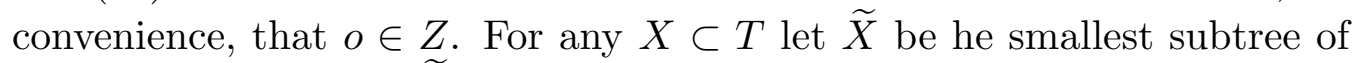
$T$ containing $X$; i.e., $\widetilde{X}=\{w \in T: w \leq x$, for some $x \in X\}$. We now proceed inductively. Let $z_{0}=o, \widetilde{Z}_{0}=\{o\}$. Now suppose that $Z_{n-1}$ and $\widetilde{Z}_{n-1}$ have already been formed. Pick $z_{n} \in Z_{n-1}^{c}$, the relative complement of $Z_{n-1}$, such that

$$
d_{\rho}\left(o, z_{n}\right)=\min \left\{d_{\rho}(o, z): z \in Z_{n-1}^{c}\right\} .
$$

Set $Z_{n}=Z_{n-1} \cup\left\{z_{n}\right\}$. One easily verifies that $Z_{n} \subseteq Z_{n+1}, \bigcup_{n \geq 0} Z_{n}=Z$, and $\bigcup_{n \geq 0} \widetilde{Z}_{n}=\widetilde{Z}$.

Let $\xi_{n}=\max \left\{x \in \widetilde{Z}_{n-1}: x \leq z_{n}\right\}$. The map $z_{n} \longmapsto \xi_{n}=\gamma\left(z_{n}\right)$ defines a function $\gamma: Z \longrightarrow T$. We call $\gamma(z)$ the landing point of $z$ on $\widetilde{Z}$. We denote by $\gamma_{+}(z)$ the immediate successor of $\gamma(z)$ on the geodesic $[\gamma(z), z]$.

Lemma 27. Let $A$ be the constant in (53). Then

$$
d_{\rho}\left(z, \gamma_{+}(z)\right) \geq(2 A)^{-1} d_{\rho}(z) .
$$

Proof. (60) is obvious for $z=o$. Otherwise, $z=z_{n}$ for some $n$ and $\gamma(z) \in\left[0, z_{j}\right]$ for some $j<n$. By the construction of $Z_{n}, d_{\rho}\left(z_{n}\right) \geq d_{\rho}\left(z_{j}\right)$. Hence,

$$
\begin{aligned}
2 d_{\rho}\left(z_{n}, \gamma_{+}\left(z_{n}\right)\right) & \geq d_{\rho}\left(z_{n}, \gamma_{+}\left(z_{n}\right)\right)+d_{\rho}\left(\gamma_{+}\left(z_{n}\right), z_{j}\right) \\
& =d_{+}\left(z_{n}, z_{j}\right)-\rho\left(z_{n} \wedge z_{j}\right)^{1-p^{\prime}} \\
& \geq A^{-1} d_{\rho}\left(z_{n}\right)
\end{aligned}
$$


Let $z \in Z$. $\phi_{z}$ will constructed by an algorithm.

Proof. For $w \in Z, w \neq o$, consider the geodesic segment $\left[\gamma_{+}(w), w\right]$. We divide $\left[\gamma^{+}(w), w\right]=a(w) \cup b(w)$ into two geodesic segments $a(w)=$ $\left[\gamma^{+}(w), \lambda(w)\right], b(w)=[\lambda(w), w]$, such that

$$
\frac{d_{\rho}\left(\gamma_{+}(w), \lambda(w)\right)}{d_{\rho}\left(\gamma_{+}(w), w\right)} \geq \frac{1}{2} \quad \text { and } \quad \frac{d_{\rho}(\lambda(w), w)}{d_{\rho}\left(\gamma_{+}(w), w\right)} \geq \frac{1}{2}
$$

This is always possible. Observe that $a(w)$ and $b(w)$ have just the point $\lambda(w)$ in common.

By construction,

$$
\widetilde{Z}=\{o\} \cup\left(\bigcup_{w \neq o, w \in Z}(a(w) \cup b(w))\right) .
$$

Set $\phi_{z}=0$ on $[o, \lambda(z))$ and let $\phi_{z}=d_{\rho}(\lambda(z), z)^{-1}$ on $b(z)$. Hence

$$
\mathcal{I} \phi_{z}(z)=1 \text {. }
$$

Suppose that $w_{1} \in Z$ is such that $\gamma\left(w_{1}\right) \in b(z)$. Let $\phi_{z}=\alpha_{1}$ on $a\left(w_{1}\right)$, where $\alpha_{1}$ is chosen so that

$$
\alpha_{1} d_{\rho}\left(\gamma_{+}\left(w_{1}\right), \lambda\left(w_{1}\right)\right)+\mathcal{I} \phi_{z}\left(\gamma\left(w_{1}\right)\right)=0
$$

Thus

$$
\mathcal{I} \phi_{z}\left(w_{1}\right)=0 \text {. }
$$

Assume now that $\phi_{z}$ has been defined on

$$
W_{n-1}(z)=b(z) \cup a\left(w_{1}\right) \cup \cdots \cup a\left(w_{n-1}\right),
$$

a subtree of $T$, in such a way that

$$
\mathcal{I} \phi_{z}(z)=1, \quad \mathcal{I} \phi_{z}\left(w_{1}\right)=\cdots=\mathcal{I} \phi_{z}\left(w_{n-1}\right)=0 .
$$

If $w_{n} \in Z \backslash\left\{z, w_{1}, \ldots, w_{n-1}\right\}$ lands on $W_{n-1}(z)$, i.e. $\gamma\left(w_{n}\right) \in W_{n-1}(z)$, then define $\phi_{z}=\alpha_{n}$ on $a\left(w_{n}\right)$, where

$$
\alpha_{n} d_{\rho}\left(\gamma_{+}\left(w_{n}\right), \lambda\left(w_{n}\right)\right)+\mathcal{I} \phi_{z}\left(\gamma\left(w_{n}\right)\right)=0 .
$$

As a consequence, $\mathcal{I} \phi_{z}\left(w_{n}\right)=0$. 
Iterate the procedure. Observe that it could run for infinitely many $n$. If $x \in T$ and $\phi_{z}(x)$ has not been defined by the algorithm then set $\phi_{z}(x)=0$. By (63) for $n=0,1, \ldots$, we have that $\mathcal{I} \phi_{z}(w)=\delta_{z}(w), w \in Z$, which is (56).

Now consider the intersection of the supports of $\phi_{z}$ and $\phi_{w}, z \neq w$. First note that if $\operatorname{supp}\left(\phi_{z}\right) \cap \operatorname{supp}\left(\phi_{w}\right) \neq \varnothing$, then the intersection consists of one point, which is either $\lambda(z)$ or $\lambda(w)$. To see this let $z=z_{n}$, where the indexing is that given by (59). If $w=z_{m}, m>n$, and $\operatorname{supp}\left(\phi_{z}\right) \cap$ $\operatorname{supp}\left(\phi_{w}\right) \neq \varnothing$, then $w$ lands on the support of $\phi_{z}$ and the intersection of the two supports is $x=\lambda(w)$. Now note that $x$ in $T$ cannot also be the landing point $\lambda(\xi)$ for some other $\xi \in Z$. If a third point $\xi=z_{l}, l>m, n$, were such that $\lambda(\xi)=\lambda(w)$, then $\xi$ would land on the support of $\phi_{w}$ and so,

$$
\lambda(\xi)>\gamma(\xi)=\lambda(w)=\lambda(\xi)
$$

which is absurd. Combining these observations shows that no $x \in T$ is in the support of more than two $\phi_{z}$, as required for (58).

In order to obtain the estimate (57) for $\left\|\phi_{z}\right\|_{L^{p}(\rho)}$, we need two more lemmas.

Lemma 28. There is a constant $C>0$ such that, if $z \in Z, x \in(\gamma(w), w]$, $w>\lambda(z)$, then

$$
\left|\phi_{z}(x)\right| \leq C d_{\rho}(w)^{-1}
$$

Also, $\phi_{z}(x)=0$ otherwise.

Proof of the Lemma. By construction, $\phi_{z}$ is monotone on sets of the form $(\gamma(w), w]$. An induction argument then shows that $0 \leq \phi_{z}(x) \leq 1$. Thus, by

$$
\left|\phi_{z}(x)\right| \leq d_{\rho}\left(\gamma_{+}(w), \lambda(w)\right)^{-1} \sim d(w, \gamma(w)) \leq d(w)^{-1}
$$

if $x \in(\gamma(w), w]$, by Lemma 27. The same reasoning applies if $x \in(\gamma(z), z]$.

The other $x$ 's are never considered in the procedure for constructing $\phi_{z}$, hence for them $\phi_{z}(x)=0$.

Lemma 29. If $\nu_{Z}$ is $p$-Carleson, then there is $C>0$ such that, for all $x \in T$,

$$
\sum_{z \in S(x) \cap Z} d_{\rho}(z)^{1-p} \leq C d_{\rho}(x)^{1-p}
$$


Proof of the Lemma. This lemma is a specialization of Proposition 8 to the case of $\mu=\nu_{Z}$.

From Lemma 28 and Lemma 29

$$
\begin{aligned}
\left\|\phi_{z}\right\|_{L^{p}(\rho)}^{p} & =\sum_{\substack{w \geq \lambda(z) \\
w \in Z}} \sum_{\substack { w \in(\gamma(w), w] \\
\begin{subarray}{c}{w \geq \lambda(z) \\
w \in Z{ w \in ( \gamma ( w ) , w ] \\
\begin{subarray} { c } { w \geq \lambda ( z ) \\
w \in Z } }\end{subarray}}\left|\phi_{z}(x)\right|^{p} \rho(x) \\
& \leq C \sum_{\substack{w \\
\rho}} \frac{d_{\rho}(w, \gamma(w))}{d_{\rho}(w)^{p}} \\
& \leq C d_{\rho}(\nu(z))^{1-p} \\
& \leq C d_{\rho}(z)^{1-p}
\end{aligned}
$$

where the last inequality follows from Lemma 27. This shows the second statement in (57), hence the theorem.

\section{Interpolating sequences in analytic Besov spaces.}

In this section we consider results about interpolating sequences for the analytic Besov spaces. For simplicity, we only consider unweighted Besov spaces. First we consider the case of $B_{2}$, where the problem was partially solved by Bishop and, independently, was completely solved by Marshall and Sundberg. In Theorem 31, we characterize those Cantor-like sequences that are interpolating for $B_{2}$. For $p \in(1, \infty)$, we examine some sequences with a high degree of symmetry in Theorem 33. The results we obtain for $B_{2}$ are sharper than those we obtain for $p \neq 2$. In particular, we think that Theorem 33 is off target for $p>2$.

(After this manuscript was completed we received a preprint from B. Böe [Boe] in which he has solved the analogous problem in which he characterizes the interpolating sequences for $B_{p}, 1<p<\infty$.)

For $1 \leq p<\infty$ and $Z$ a sequence in $\mathbb{D}$, we say that $Z$ is interpolating for $B_{p}$ if the operator $T_{p}$ defined for $z_{a} \in Z$ by

$$
T_{p} f\left(z_{a}\right)=\left(1+\log \frac{1}{1-\left|z_{a}\right|^{2}}\right)^{-1 / p^{\prime}} f\left(z_{a}\right)
$$

is a bounded map from $B_{p}$ onto $L^{p}(Z)$. The normalizing factor in (67) insures $T_{p}$ maps $B_{p}$ to $L^{\infty}(Z)$. 
Lemma 30. ([Zhu, Exercise 5.17]). If $f \in B_{p}$, then $f$ is Hölder continuous with exponent $p^{-1}$ with respect to the hyperbolic metric. In fact

$$
|f(z)-f(w)| \leq C\|f\|_{B_{p}^{*}} d_{h}(z, w)^{1 / p^{\prime}} .
$$

7.1. $p=2$.

Bishop and Marshall-Sundberg have proved the following:

Theorem BMS ([MS]). A sequence $Z \subset \mathbb{D}$ is interpolating for $B_{2}$ if and only if the following two conditions hold

1) The measure $\mu_{Z}$ given by

$$
\mu_{Z}=\sum_{z_{a} \in Z}\left(1+\log \frac{1}{1-\left|z_{a}\right|^{2}}\right)^{-1} \delta_{z_{a}}
$$

is Carleson for $B_{2}$.

2) There are $A, B>0$ so that whenever $z_{a} \neq z_{b}$ are points in $Z$

$$
d\left(0, z_{a}\right) \leq A d\left(z_{a}, z_{b}\right)+B .
$$

This separation condition (70) is of the same form as the condition (54) which we saw in the discrete case. There are a number of equivalent reformulations of these conditions. It is easy to check that one gets equivalent conditions if, say, the left hand side of (70) is replaced by the more symmetric $d\left(0, z_{a}\right)+d\left(0, z_{b}\right)$, or if the origin (or the root) is replaced with an alternative distinguished reference point. In [MS] the condition is presented in the following form. Recall the pseudohyperbolic distance on $\mathbb{D}$ which is defined by

$$
\rho(z, w)=\left|\frac{z-w}{1-\bar{w} z}\right| .
$$

Then (70) is equivalent to there being a constant $\gamma>0$ so that whenever $z_{a} \neq z_{b}$ are points in $Z$

$$
1-\rho^{2}\left(z_{n}, z_{m}\right) \leq\left(1-\rho^{2}\left(0, z_{m}\right)\right)^{\gamma} .
$$

This equivalence of this and (70) follows from the relation between the hyperbolic and pseudohyperbolic distances and straightforward estimates. Marshall and Sundberg also offer another, intriguing, condition which is 
again equivalent, but where the equivalence, although again elementary, is less straightforward. Recall that the reproducing kernel for the Dirichlet space is given by

$$
k_{z}(w)=1+\log \frac{1}{1-w \bar{z}}
$$

It follows that for each $z, \widetilde{k}_{z}=k_{z}(z)^{-1 / 2} k_{z}$ is a unit vector in $B_{2}$. Hence the inner product of any two such functions has modulus at most one. [MS, Lemma 19] states that (70) is equivalent to the following: there is a $\sigma<1$ so that whenever $z_{a} \neq z_{b}$ are points in $Z$

$$
\left|\left\langle\widetilde{k}_{z_{a}}, \widetilde{k}_{z_{b}}\right\rangle\right| \leq \sigma
$$

The analysis in [MS] uses Stegenga's capacitary characterization of Carleson measures. It is our hope that our alternative description can be used to give an alternative, easier, approach; and one which extends to $p \neq 2$. However we have not yet been able to do that. For now, we only have partial results. We work with Cantor-like sequences because they are very well suited for an approach through trees and also because, from Stegenga's pioneering paper, they are a recurrent theme in counterexamples in the theory of the analytic Besov spaces [Ste], [MS], [Wang]. See [A] for an approach based on the explicit estimation of capacities.

Let $\left\{d_{n}: n \geq 0\right\}$ be an increasing sequence in $[0,1), d_{0}=0$. We construct a sequence $Z$ as follows

$$
Z=\left\{z(n, m): n \geq 0,1 \leq m \leq 2^{m}\right\}
$$

where

$$
|z(n, m)|=R_{n}=\left(1-e^{1-d_{n}}\right)^{1 / 2}
$$

and $\arg (z(n, m))$ is defined by the following procedure: Let $I_{0}=[0, \pi]$ and, for $n \geq 1$, let $I_{1}^{n}, \ldots, I_{2^{n}}^{n}$ be the intervals at the $n^{\text {th }}$ stage in the construction of a Cantor set in which the initial segment is $I_{0}$ and

$$
m\left(I_{j}^{n}\right)=\pi\left(1-R_{n}\right), \quad j=1, \ldots, 2^{n} .
$$

Let $\arg (z(n, 1))<\cdots<\arg \left(z\left(n, 2^{n}\right)\right)$ be the centers of such intervals.

Theorem 31. Let $Z$ be a Cantor-like sequence just described. $Z$ is an interpolating sequence if and only if, for some $C>0$ independent of $N$,

$$
\sum_{n \geq N} 2^{n} d_{n}^{-1} \leq C 2^{N} d_{N}^{-1}
$$


and, for some $\varepsilon>0$ independent of $n$,

$$
d_{n+1} \geq(1+\varepsilon) d_{n}
$$

We begin by showing that (71) completely solves the problem of the boundedness of $\mathcal{T}$.

Proposition 32. $\mathcal{T}$ is bounded if and only if (71) holds.

Proof. Recall the tree $T_{2}$ considered in Section 3. Let $\widetilde{Z} \subset T_{2}$ be a sequence so that $\alpha=(n, m) \in \widetilde{Z}$ if there is $z \in Z$ with $z \in \Delta_{\alpha}$.

The following assertions are elementary.

a) Except at the origin, $\widetilde{Z}$ is a tree-like sequence in $T_{2}$, having the structure of a homogeneous tree of degree 2 ,

b) Denote by $d$ distance in $T_{2}$ and by $d_{h}$ the hyperbolic distance on $\mathbb{D}$. We have, recalling the notation $d_{\rho}$ introduced just before Proposition 10 ,

$$
\begin{aligned}
d_{n} & \sim 1+\log \frac{1}{1-|z(n, m)|^{2}} \\
& \sim d_{h}(0, z(n, m))+1 \\
& \sim d((n, m), o)+1 \\
& \sim d_{1}((n, m)) .
\end{aligned}
$$

It follows from b) that in $(71)$ we can replace $d_{n}$ by $d((n, \cdot))=d((n, \cdot)+1)$ by changing the constant $C$. Thus, by a) and Proposition 10, we have that (71) holds if and only if $\mu_{\widetilde{Z}}=\sum_{z \in \widetilde{Z}} d(z)^{-1} \delta_{z}$ is Carleson for $(\mathcal{I}, 2)$. In turn, this is equivalent to the fact that $\mathcal{T}$ is bounded, by Proposition 5 and by b) once again.

Proof of the Theorem. Given Proposition 32 and Theorem BMS, it remains to show that (72) is equivalent to condition ii). This easily follows from an estimate of the hyperbolic distance between two points $z(n, m)$ and $z(n+1, l)$ and the fact that if either ii) or (72) hold then

$$
|\arg (z(n, m))-\arg (z(n, m+1))|
$$

is large if compared with $e^{-d_{n}}$. 
7.2. $1<p<\infty$.

We now discuss some applications of Theorem 1 and Proposition 5 to the determination of interpolating sequences for $B_{p}, 1<p<\infty$. We work with sequences that are "radially symmetric" in a sense that will be clear below. We use a Neumann series argument introduced, in the study of interpolating sequences for the Bergman space, by R. Rochberg in $[R]$. See also [Wang] for results for the Besov spaces obtained by a similar argument.

Let $\lambda>1$ and $c_{0}>0$ be fixed. Consider a symmetric sequence $Z \subset \mathbb{D}$ given by

$$
Z=\left\{z(n, m): n \geq 0,1 \leq m \leq 2^{n}\right\}
$$

where

$$
z(n, m)=R_{n} e^{i \theta_{n, m}}, \quad 1 \leq m \leq 2^{n}
$$

and

$$
R_{n}=\left(1-e^{-c_{0} \lambda^{n}}\right)^{1 / 2}, \quad \theta_{n, m}=2^{-n} 2 \pi\left(m+\frac{1}{2}\right) .
$$

For fixed $n \geq 0$, there are $2^{n}$ points of $Z$ having Euclidean distance $R_{n}$ from the center of $\mathbb{D}$ and they are equally spaced on the circle $\left\{|z|=R_{n}\right\}$.

Theorem 33. Let $Z$ be the symmetric sequence defined in (73).

1) Let $1<p \leq 2$. If

$$
\lambda>2^{1 /(p-1)}
$$

and $c_{0}=c_{0}(p, \lambda)$ is large enough, then $Z$ is interpolating for $B_{p}$.

2) Let $2<p$. If

$$
\lambda>2^{1 /\left(p^{\prime}-1\right)}
$$

and $c_{0}=c_{0}(p, C)$ is large enough, then $Z$ is interpolating for $B_{p}$.

3) If $Z$ is interpolating for $B_{p}$, then $\lambda>2^{1 /(p-1)}$.

For comparison with the examples at the end of Section 3 note that (74) is equivalent to

$$
p>1+\frac{\log 2}{\log \lambda} .
$$

The theorem is relatively sharp in the range $1<p \leq 2$. For $p=2$, using Theorem BMS and Proposition 34 below, it is easy to see that the sequence 
$Z$ in 1 ) is, in fact, interpolating for all $c_{0}>0$. We think that this is the case for all $p$. We suspect the result for $p>2$ is less sharp and we discuss that briefly after the proof.

The proof of 1) and 2) relies on the construction of a right inverse for $T_{p}$, i.e. of a bounded, linear map $R: L^{p}(Z) \longrightarrow B_{p}$ such that $T_{p} \circ R=\mathrm{Id}$. Hence, the solution $f \in B_{p}$ that we provide for the equation

$$
T_{p} f=g, \quad g \in L^{p}(Z)
$$

is linear. The solution of the interpolating problem given in [MS] is indirect and we do not know if it implicitly contains a linear construction.

The first step in the proof of Theorem 33 is the characterization of those $\lambda$ for which $T_{p}$ is bounded.

Proposition 34. Let $1<p<\infty$ and let $Z$ as above. $T_{p}$ is bounded from $B_{p}$ to $L^{p}(Z)$ if and only if (74) holds.

Proof. Observe first that, by definition, $T_{p}$ is bounded from $B_{p}$ to $L^{p}(Z)$ if and only if the measure

$$
\mu_{Z}=\sum_{z_{a} \in Z}\left(1+\log \frac{1}{1-\left|z_{a}\right|^{2}}\right)^{1-p} \delta_{z_{a}}
$$

is a Carleson measure for $B_{p}$. By Theorem 1 and Proposition 5, we can discretize $\mu_{Z}$ and study an analogous problem on $T_{2}$, the tree defined in (8).

Consider the sequence $Z^{\prime} \subset T_{2}$ defined as follows. $\xi_{a} \in Z^{\prime}$ if and only if there exists $z_{a} \in Z$ such that $z_{a} \in \Delta_{\xi_{a}}$. If $\left|z_{a}\right|=R_{n}$, by an estimate of the hyperbolic distance and the properties of the Whitney decomposition,

$$
1+c_{0} \lambda^{n}=1+\log \frac{1}{1-\left|z_{a}\right|^{2}} \sim 1+d_{h}\left(z_{a}, 0\right) \sim 1+d\left(\xi_{a}, o\right) .
$$

As a consequence, $\sharp\left\{z_{a} \in Z: z_{a} \in \Delta_{\alpha}\right\}$ is bounded by a constant independent of $\alpha \in T_{2}$. These estimates also show that $d\left(\xi_{a}\right)^{1-p}$ is comparable with $\mu_{Z}\left(\Delta_{\xi_{a}}\right)$. Hence, by Proposition $5, \mu_{Z}$ is $p$-Carleson if and only if the measure

$$
\nu_{Z}=\sum_{\xi_{a} \in Z^{\prime}} d\left(\xi_{a}\right)^{1-p} \delta_{\xi_{a}}
$$

is Carleson for $(\mathcal{I}, p)$. 
If $\nu_{Z}$ is Carleson for $(\mathcal{I}, p)$ then it is bounded and hence

$$
\infty>\nu_{Z}\left(T_{2}\right) \sim \sum_{n \geq 0} 2^{n} \lambda^{(1-p) n} .
$$

This forces $\lambda>2^{1 /(p-1)}$.

Suppose, conversely, that $\lambda>2^{1 /(p-1)}$. To show that $\nu_{Z}$ is Carleson for $(\mathcal{I}, p)$ we verify that it satisfies (6). Let $\beta \in T_{2}$ and let $\xi(\beta)$ be an element $\xi$ in $Z^{\prime} \cap S(\beta)$ such that $d(\xi)$ is minimum. We claim that

$$
\nu_{Z}(S(\beta)) \sim d(\xi(\beta))^{1-p} .
$$

Then, if $\alpha \in T_{2}$,

$$
\begin{aligned}
\sum_{\beta \in S(\alpha)}\left|\nu_{Z}(S(\beta))\right|^{p^{\prime}} & \leq C \sum_{\beta \in S(\alpha)} d(\xi(\beta))^{(1-p) p^{\prime}} \\
& =C \sum_{\xi \in S(\alpha) \cap Z^{\prime}} \sharp\{\beta \in S(\alpha): \xi=\xi(\beta)\} d(\xi(\beta))^{(1-p) p^{\prime}} \\
& \leq C \sum_{\xi \in S(\alpha) \cap Z^{\prime}} d(\xi)^{1-p} \\
& =C \nu_{Z}(S(\alpha)) .
\end{aligned}
$$

Hence (78) holds and the proposition is proved modulo the claim.

We only have to verify (78). Suppose that $d(\beta, o)=k$. Then

$$
\bigcup_{\gamma \in S(\beta)} \Delta_{\gamma}=\left\{z \in \mathbb{D}: 1-2^{-k}<|z|<1, \theta(\beta)<\arg (z) \leq \theta(\beta)+\frac{2 \pi}{2^{k}}\right\},
$$

where $\theta(\beta)$ is an angle depending on $\beta$, by (8). Fix $n \geq 0$ and consider $Z_{n}$, the set of those $\xi \in Z^{\prime}$ corresponding to some $z_{a} \in Z$ with $\left|z_{a}\right|=R_{n}$. The terms of $Z$ corresponding to a fixed $n$ are uniformly spaced. Hence, if $R_{n} \geq 1-2^{-k}$ then $\left[2^{n-k}\right] \leq \sharp\left\{\xi \in Z_{n} \cap S(\beta)\right\} \leq\left[2^{n-k}\right]+1$. Now, $R_{n} \geq 1-2^{-k}$ if $n>C_{1} \log k+C_{2}$, where $C_{1}$ and $C_{2}$ are positive constants. If $k$ is large enough, we can find $n$ so that $C_{1} \log k+C_{2} \leq n \leq k$. In that case $\xi(\beta)$ is unique and it will correspond to $z_{a_{0}}$ with $\left|z_{a_{0}}\right|=R_{n_{0}}$, where $n_{0}$ is one of such $n$ 's. We can now estimate

$$
\begin{aligned}
\nu_{Z}(S(\beta)) & \leq C \sum_{n \geq n_{0}}\left(\left[2^{n-k}\right]+1\right) \lambda^{(1-p) n} \\
& \leq C \sum_{n \geq n_{0}} 2^{n-n_{0}} \lambda^{(1-p) n} \\
& \leq C \lambda^{(1-p) n_{0}} \\
& \leq C d(\xi(\beta))^{1-p} .
\end{aligned}
$$


This shows (78) for $k \geq k_{0}$. By increasing the constant we can force (78) for all $k$.

Proof of Theorem 33. (3) follows immediately from the only if part of Proposition 34.

To prove 1) and 2), we construct a right inverse $R$ for $T_{p}$. We look for a map

$$
R_{0}: L^{p}(Z) \longrightarrow B_{p}
$$

such that

$$
\left\|T_{p} \circ R_{0}-\operatorname{Id}\right\|_{L^{p}(Z)}<1
$$

where Id is the identity. Then, $T_{p} \circ R_{0}$ is invertible and $R=R_{0} \circ\left(T_{p} \circ R_{0}\right)^{-1}$ is a right inverse for $T_{p}$.

We define $R_{0}$ acting on sequences

$$
\mathbf{a}=\left\{a_{n, m}: n \geq 0,1 \leq m \leq 2^{n}\right\} \in L^{p}(Z)
$$

by the formula

$$
R_{0}(\mathbf{a})(z)=\sum_{\substack{n \geq 0 \\ 1 \leq m \leq 2^{n}}} a_{n, m} f_{n, m}(z)
$$

where

$$
f_{n, m}(z)=\left(1+\log \frac{1}{1-\left|z_{n, m}\right|^{2}}\right)^{-1 / p}\left(1+\log \frac{1}{1-\bar{z}_{n, m} z}\right) .
$$

This is a reasonable guess for an approximate right inverse for $T_{p}$, since the function

$$
\phi_{n, m}(z)=1+\log \frac{1}{1-\bar{z}_{n, m} z}
$$

is the reproducing kernel of $B_{p}$ at $z_{n, m}$ and $\left\|f_{n, m}\right\|_{B_{p}} \sim 1$ independently of $n, m$.

One easily verifies that $R_{0}^{*}$, the adjoint of $R_{0}$, is the map of $B_{p^{\prime}}$ to $L^{p^{\prime}}(Z)$ given by

$$
R_{0}^{*} f=T_{p^{\prime}} f .
$$

Our assumptions (74) and (75) in the theorem imply, by Proposition 34, that $R_{0}=T_{p^{\prime}}^{*}$ is bounded from $L^{p}(Z)$ to $B_{p}$. 
The operator $T_{p} \circ R_{0}: L^{p}(Z) \longrightarrow L^{p}(Z)$ is given by

$$
T_{p} \circ R_{0}(\mathbf{a})(n, m)=\sum_{\substack{j \geq 0 \\ 1 \leq i \leq 2^{j}}} a_{j, i} \frac{f_{i, j}\left(z_{n, m}\right)}{\left(1+\log \frac{1}{1-\left|z_{n, m}\right|^{2}}\right)^{1 / p^{\prime}}} .
$$

Thus,

$$
\left(T_{p} \circ R_{0}-\mathrm{Id}\right)(\mathbf{a})(n, m)=\sum_{\substack{j \geq 0 \\ 1 \leq i \leq 2^{j} \\(j, i) \neq(n, m)}} A((n, m),(j, i)) a(j, i)
$$

where

$$
\begin{aligned}
& A((n, m),(j, i)) \\
& \qquad=\frac{1+\log \frac{1}{1-\bar{z}_{j, i} z_{n, m}}}{\left(1+\log \frac{1}{1-\left|z_{n, m}\right|^{2}}\right)^{1 / p^{\prime}}\left(1+\log \frac{1}{1-\left|z_{j, i}\right|^{2}}\right)^{1 / p}} .
\end{aligned}
$$

In order to verify that (79) holds, we need an estimate for $A((n, m),(j, i))$.

Lemma 35. There exists $C=C(\lambda)>0$ independent of $c_{0}$ such that

$$
A((n, m),(j, i)) \leq \frac{C}{c_{0}} \frac{\max \{n, j\}}{\lambda^{n / p^{\prime}+j / p}}
$$

Proof of the Lemma. We denote by $C$ a constant that does not depend on $c_{0}$. Let $z=R e^{i \theta}, 0<R<1,|\theta| \leq \pi$. Then, $\left|1-R e^{i \theta}\right|^{2}=(1-R)^{2}+$ $2 R(1-\cos \theta)$, and hence $\left|1-R e^{i \theta}\right| \geq C R^{1 / 2} \theta$. Now, $\overline{z_{j, i}} z_{n, m}=R e^{i \theta}$, with $\theta \geq \pi \min \left\{2^{-j}, 2^{-n}\right\}$ and $R \geq 1-e^{-c_{0} d_{1}}$ if $n, j \geq 0,(n, m) \neq(j, i)$. Thus,

$$
\begin{aligned}
\left|1+\log \frac{1}{1-\overline{z_{j, i}} z_{n, m}}\right| & \leq C+\log \left|\frac{1}{1-\overline{z_{j, i}} z_{n, m}}\right| \\
& \leq C\left(\max \{n, j\}+\log \frac{1}{1-e^{-c_{0} d_{1}}}\right) .
\end{aligned}
$$

Estimate (84) follows. 
In order to prove (79), we first show that it suffices to test

$$
\mathcal{A}=T_{p} \circ R_{0}-\mathrm{Id}
$$

on spherically symmetric sequences; those $\mathbf{a}_{\mathbf{s}} \in L^{p}(Z)$ such that $a_{s}(n, m)=$ $a_{s}(n)$ is independent of $m$. To see this, let $\mathbf{a} \in L^{p}(Z)$ and define

$$
a_{s}(n)=a_{s}(n, m)=\frac{1}{2^{n}} \sum_{1}^{2^{n}} a(n, m)
$$

Then,

$$
\mathcal{A} a=\mathcal{A} a_{s} \quad \text { and } \quad\left\|a_{s}\right\|_{L^{p}(Z)} \leq\|a\|_{L^{p}(Z)} .
$$

The first assertion is a straightforward calculation, while the second follows from Jensen's inequality. By (85),

$$
\frac{\|\mathcal{A} a\|_{L^{p}(Z)}}{\|a\|_{L^{p}(Z)}} \leq \frac{\left\|\mathcal{A} a_{s}\right\|_{L^{p}(Z)}}{\left\|a_{s}\right\|_{L^{p}(Z)}}
$$

as claimed.

Now let $\mathbf{a}=\mathbf{a}_{\mathbf{s}}$ be a radially symmetric sequence.

$$
\begin{aligned}
&\|\mathcal{A} \mathbf{a}\|_{L^{p}(Z)}^{p}=\sum_{n, m}\left|\sum_{j, i} A((n, m),(j, i)) a(j)\right|^{p} \\
& \operatorname{by}(84) \leq C c_{0}^{-p} \sum_{n} 2^{n}\left|\sum_{j, i} \max \{n, j\} \lambda^{-\left(n / p^{\prime}+j / p\right)} a(j)\right|^{p} \\
&=C c_{0}^{-p} \sum_{n} 2^{n} \lambda^{(1-p) n}\left|\sum_{j} 2^{j} \max \{n, j\} \lambda^{-j / p} a(j)\right|^{p} \\
& \leq C c_{0}^{-p} \sum_{n} 2^{n} \lambda^{(1-p) n}\left(\sum_{j} 2^{j}|a(j)|^{p}\right) \\
& \cdot\left(\sum_{j} 2^{j} \lambda^{\left(1-p^{\prime}\right) j} \max \{n, j\}^{p^{\prime}}\right)^{p / p^{\prime}}
\end{aligned}
$$

by Hölder's inequality $\leq C\|\mathbf{a}\|_{L^{p}(Z)}^{p} c_{0}^{-p} \sum_{n} 2^{n} \lambda^{(1-p) n} n^{p}$

$$
\cdot\left(\sum_{j} 2^{j} \lambda^{\left(1-p^{\prime}\right) j} j^{p^{\prime}}\right)^{p / p^{\prime}} .
$$


By (74), (75) and properties of the geometric series,

$$
\sum_{n} 2^{n} \lambda^{(1-p) n} n^{p}\left(\sum_{j} 2^{j} \lambda^{\left(1-p^{\prime}\right) j} j^{p^{\prime}}\right)^{p / p^{\prime}} \leq C(\lambda) .
$$

Thus (79) holds if $c_{0}$ is large enough.

Corollary 36. If the assumptions of Theorem 33, 1), 2) hold then $T_{p}$ has a linear the right inverse.

Using our proof we can prove that a sequence is interpolating for $B_{p}$ if and only if we can prove it is interpolating for $B_{p^{\prime}}$. Specifically, we use estimates for

$$
\left\|T_{p} \circ R_{0}-\mathrm{Id}\right\|_{L^{p}(Z)}=\left\|T_{p^{\prime}} \circ T_{p}^{*}-\operatorname{Id}\right\|_{L^{p^{\prime}}(Z)} .
$$

Hence, if our proof that $Z$ is interpolating works for $p$, it holds for $p^{\prime}$ as well. This is why the sufficient conditions in Theorem 33 involve $\min \left\{p, p^{\prime}\right\}$ and hence has a different appearance for $1<p \leq 2$ and $p>2$. This is in contrast with Theorem 26 about interpolating sequences for $(\mathcal{I}, p)$, which is proved by different methods.

In [Wang], Wang presents a necessary conditions for a sequence to be interpolating for $B_{p}$, He conjectured that the condition is sufficient as well.

Theorem W ([Wang]). If a sequence $Z \subset \mathbb{D}$ is interpolating for $B_{p}$, then

1) $\mu_{Z}$ is a Carleson measure for $B_{p}$ and

2) there are $A, B>0$ so that whenever $z_{a} \neq z_{b}$ are points in $Z$

$$
d\left(0, z_{a}\right) \leq A d\left(z_{a}, z_{b}\right)+B
$$

If $1<p \leq 2$, the sequences that we consider in Theorem 33 are interpolating only if they satisfy the conditions of Theorem $\mathrm{W}$, and they are interpolating if they satisfy the conditions and $c_{0}$ is large enough. This fact goes in the direction of Wang's conjecture. On the other hand, by Proposition 34, if Wang's conjecture were true, the sequences we consider in Theorem 33 would be interpolating under the assumption (74), even in the case $2 \leq p$. (The result of $\mathrm{B}$. Böe mentioned at the beginning of this section shows that this conjecture is correct.) 


\section{Carleson measures for Hardy spaces on trees.}

As we have mentioned, the Carleson measures for the Hardy space $H^{2}=B_{2}\left(1-|z|^{2}\right)$ are characterized by the single box condition (37) (with $\alpha=1)$. That condition is strictly weaker than condition (3), the appropriate examples are given, for instance, in the proof of Theorem 11. In this section we see that those facts have exact analogs in the discrete context.

First, in analogy with the continuous case, we are going to define $H^{2}(T)$, the Hardy space of harmonic functions on the dyadic tree, $T$. Next, we show that the Hardy space can be characterized as the space of those harmonic functions $\Phi$ on $T$ such that $\Phi=\mathcal{I} \varphi$ and $\varphi$ belongs to $L^{2}(\rho)$, where $\rho$ is a suitable weight on $T$. We then show that the Carleson measures for $H^{2}(T)$ can be characterized by a single box condition analogous to the one used for the classical Hardy space. We then observe that this condition is not equivalent to our condition (6). More precisely, there are measures $\mu$ on $T$ that are Carleson for $H^{2}(T)$, but that are not Carleson for $(\mathcal{I}, \rho, p)$.

This shows that for some weights adding structure to the functions (i.e., harmonicity) affects the set of the Carleson measures in the discrete case just as it does in the continuous case. It is easy to prove similar results concerning the Carleson measures for the weighted Bergman spaces on trees.

Let $T=T_{2}$ be the dyadic tree, with root $o$. We say that $x, y \in T$ are adjacent, $x \sim y$, if $d(x, y)=1$. A function $\Phi$ on $T$ is harmonic if it satisfies the mean value property

$$
\Phi(x)=\frac{1}{3} \sum_{y \sim x} \Phi(y)
$$

(with 3 replaced by 2 if $x=o$.) The tree $T$ has a boundary, $\Omega$, which is defined as follows. $\Omega$ is the set of all infinite geodesics $\omega=\left(\omega_{0}, \omega_{1}, \ldots, \omega_{n} \ldots\right)$ starting at $\omega_{0}=o$. We can extend the partial order on $T$ to $\bar{T}=T \cup \Omega$ by letting $x \leq \omega$ whenever $x \in \omega$, so that $\omega$ can be thought of as a point at infinity of $T$. This way we extend the operation $x \wedge y$ to $\bar{T}$.

One could think of $(T, d)$ as of a metaphor for the unit disk in the complex plane, endowed with its hyperbolic metric, and continue the metaphor by thinking of $\Omega$ as the boundary of the disk. On $\bar{T}$ we have a Euclidean metric, $d_{e}$,

$$
d_{e}(x, x)=0, d_{e}(x, y)=\frac{2}{3} 2^{-d(x \wedge y)}, \quad \text { if } x \neq y, x, y \in \bar{T},
$$

with respect to which $T$ and $\Omega$ are compact and have diameter 1 . For $x \in T$ let $\overline{S(x)}$ be the closure of $S(x)$ with respect to $d_{e}$ and $I(x)=\overline{S(x)} \cap \Omega$. 
The sets $I(x)$ are a basis for the topology on $\Omega$ induced by $d_{e}$. On $\Omega$, there is a Borel measure

$$
m(I(x))=\frac{2}{3} 2^{-d(x, 0)}=\operatorname{diam}(I(x)) .
$$

From now on we will use the norm notation

$$
|x|=d(x, o) .
$$

Let $1 \leq p \leq \infty$. The Hardy space $H^{p}(T)$ is the set of functions $\Phi$ that are harmonic on $T$ for which

$$
\|\Phi\|_{p}=\sup _{n \geq 1}\left(\frac{1}{2^{n}} \sum_{d(x, o)=n}|\Phi(x)|^{p}\right)^{1 / p}<\infty .
$$

From now on we only consider $1<p<\infty$. It is well known that

$$
\Phi(\omega)=\lim _{n \rightarrow \infty} \Phi\left(\omega_{n}\right)
$$

exists $m$-almost everywhere and that

$$
\|\Phi\|_{p}^{p}=\lim _{n \rightarrow \infty} \frac{1}{2^{n}} \sum_{d(x, o)=n}|\Phi(x)|^{p}=\int_{\Omega}|\Phi(\omega)|^{p} .
$$

We say that a measure $\mu$ on $T$ is Carleson for $H^{p}(T)$ if

$$
\sum_{x \in T}|\Phi(x)|^{p} \mu(x) \leq C(\mu)\|\Phi\|_{p}^{p},
$$

for all harmonic functions $\Phi$ on $T$, with a constant $C(\mu)$ that only depends on $\mu$. The monographs [DB] or [So], and the articles [Cart], [KPT], and [T] develop the theory of the harmonic functions on trees and the associated Hardy spaces in several directions.

We characterize the Carleson measures for $H^{p}(T)$, following the proof in [St, II.2], for the classical case.

Theorem 37. Let $1<p<\infty . \mu$ is Carleson for $H^{p}(T)$ if and only if, for some $C_{1}(\mu)$ that only depends on $\mu$,

$$
\mu(S(x)) \leq C_{1}(\mu) m(I(x)) .
$$


Proof. Suppose that $\mu$ is Carleson for $H^{p}(T)$. Let $z \in T \backslash\{o\}$, and suppose that $z^{-}$is the predecessor of $z$. Define $\Phi_{z}: T \longrightarrow \mathbb{R}$ by

$$
\Phi_{z}(\xi)= \begin{cases}1-2^{-k-1}, & \text { if } \xi \geq z, d(\xi, z)=k \\ -\left(1-2^{-k-1}\right), & \text { if } \xi \wedge z=z^{-}, d\left(\xi, z^{-}\right)=k+1 \\ 0, & \text { otherwise }\end{cases}
$$

$\Phi_{z}$ is harmonic on $T$. A straightforward calculation shows that

$$
\left\|\Phi_{z}\right\|_{p}^{p} \sim 2^{-|z|}
$$

and that

$$
\sum_{x \in T}\left|\Phi_{z}(x)\right|^{p} \mu(x) \geq \frac{1}{2} \mu(S(z))
$$

hence, that (88) holds.

Proof. In the proof of the converse, it will be convenient to work with a sequence of finite trees that, in some sense, approximate $T$. Let $T_{n}=$ $\{x \in T:|x| \leq n\}$ and $\Omega_{n}=\partial T_{n}=\left\{x \in T_{n}:|x|=n\right\}$. For $x \in \Omega_{n}$, set $\lambda_{n}(x)=2^{-n} . \lambda_{n}$ is the harmonic measure on $\Omega_{n}$. If $f: \Omega_{n} \longrightarrow \mathbb{R}$, the maximal function of $f, M_{n} f: \Omega_{n} \rightarrow \mathbb{R}$, is defined by

$$
M f(x)=\sup _{o \leq z \leq x} \frac{1}{2^{n-|z|}} \sum_{y \in I_{n}(z)}|f(y)| .
$$

Here, $I_{n}(z)=\left\{y \in \Omega_{n}: y \geq z\right\}$. Standard arguments show that there exists a constant $C_{p}$ only depending on $p$, in particular independent of $n$, such that

$$
\|M f\|_{L^{p}\left(\lambda_{n}\right)} \leq C_{p}\|f\|_{L^{p}\left(\lambda_{n}\right)} .
$$

A function $\Phi$ on $T_{n}$ is harmonic if and only if its restriction to $T_{n} \backslash \Omega_{n}$ satisfies the mean value property. The following lemma says that the maximal function dominates the Poisson integral.

Lemma 38. There exists a constant $C>0$, independent of $n$, such that, if $\Phi$ is harmonic on $T_{n}$ and $f=\left.\Phi\right|_{\Omega_{n}}$,

$$
\sup _{o \leq z \leq x}|\Phi(z)| \leq M f(x) .
$$


Proof of Lemma. Harmonic functions on trees have the following probabilistic interpretation [Cart], which is analogous to Kakutani's theorem on harmonic functions in $\mathbb{R}^{n}[\mathrm{Du}]$. Let $X=\left(X_{1}, \ldots, X_{n}, \ldots\right)$ be the symmetric, one step random walk on $T$; i.e., the random particle in position $x \in T$ at time $k \geq 0$ moves with probability $1 / 3$ to each of its neighbors at time $k+1$. In terms of conditional probabilities, if $y \sim x$,

$$
\mathbb{P}\left(X_{k+1}=y \mid X_{k}=x\right)=\frac{1}{3} \text {. }
$$

Define the stopping time

$$
\tau_{n}=\min \left\{k \geq 0: X_{k} \in \Omega_{n}\right\}
$$

Then, if $|z| \leq n$ and $\Phi, f$ are as above,

$$
\Phi(z)=\mathbb{E}^{z} f\left(T_{\tau}\right)
$$

Denote the points in the geodesic $[o, z]$ from $z$ to $o$ by $o=w_{|z|}<w_{|z|-1}<$ $\cdots<w_{0}=z$. It follows from the strong Markov property of the process $X$ that there exist numbers $c_{j}>0$, depending only on $j$, such that

$$
\mathbb{E}^{z} f\left(X_{\tau}\right)=\sum_{j=0}^{|z|} c_{j}^{w_{j}} \mathbb{E}\left(f\left(X_{\tau}\right) \mid X \in S\left(w_{j}\right)\right)
$$

In fact,

$$
c_{j}=\mathbb{P}^{w_{j}}\left(X_{k} \in S\left(w_{j}\right) \text { for all } k \geq 0\right) \prod_{l=0}^{j} \mathbb{P}^{w_{l-1}}\left(\text { there exists } k: X_{k}=w_{l}\right)
$$

Taking $f \equiv 1$, since the process $X$ is transient,

$$
\sum_{j=0}^{|z|} c_{j}=1
$$

By the symmetries of $X$,

$$
\mathbb{E}^{w_{j}}\left(f\left(X_{\tau}\right) \mid X \in S\left(w_{j}\right)\right)=\frac{1}{2^{n-\left|w_{j}\right|}} \sum_{y \in I_{n}\left(w_{j}\right)} f(y) .
$$


Hence,

$$
M f(z)=\sup _{j} \frac{1}{2^{n-\left|w_{j}\right|}} \sum_{y \in I_{n}\left(w_{j}\right)}|f(y)| \geq|\Phi(z)|
$$

by (91), (92) and the fact that $\sum_{j=0}^{|z|} c_{j}=1$.

Lemma 8 can be rephrased as follows. Let $\Psi(z)=|\Phi(z)|$ and $\Theta(x)=$ $M f(x), z \in T_{n}, x \in \Omega_{n}$. Then

$$
\max _{o \leq z \leq x} \Psi(z) \leq \Theta(x)
$$

The next lemma can be proved by again following the argument of Stein in [St, II.2].

Lemma 39. If $\Psi$ and $\Theta$ are positive functions on $T_{n}$ and $\Omega_{n}$, respectively, such that (94) holds and if $\mu$ is a positive measure on $T$ such that (88) holds, then

$$
\mu\left(z \in T_{n}: \Psi(z)>\alpha\right) \leq C(\mu) \lambda_{n}\left(x \in \Omega_{n}: \Theta(x)>\alpha\right)
$$

Suppose, now, that $\mu$ satisfies (88). By Lemma 8, (94), Lemma 39 and (89) we have

$$
\sum_{|z| \leq n}|\Phi(z)|^{p} \mu(z) \leq C(\mu) \frac{1}{2^{n}} \sum_{|x|=n}|M f(x)|^{p} \leq C_{p} \frac{1}{2^{n}} \sum_{|x|=n}|\Phi(z)|^{p} .
$$

Recall that $f=f_{n}=\left.\Phi\right|_{\Omega_{n}}$. If we let $n \longrightarrow \infty$, we obtain that $\mu$ is Carleson.

For $\Phi \in H^{2}(T)$, there exists a unique $\varphi$ such that $\Phi=\mathcal{I} \varphi, \varphi(z)=$ $\Phi(z)-\Phi\left(z^{-}\right)$. We again use the notation $\varphi=D \Phi$. Let $L^{2}\left(2^{-|z|}\right)$ be the $L^{2}$ space corresponding to the measure $\nu(z)=2^{-|z|}$ on $T$.

Theorem 40. We have the following equivalence of norms on $H^{2}(T)$

$$
\|\Phi\|_{2} \sim\|D \Phi\|_{L^{2}\left(2^{-|z|}\right)}
$$

As a consequence, we have the following corollary. 
Corollary 41. There exists a measure $\mu$ on $T$ such that $\mu$ is not Carleson for $\left(\mathcal{I}, 2,2^{-|z|}\right)$, but the inequality $\|\Phi\|_{L^{2}(\mu)} \leq C\|\Phi\|_{2}$, i.e.,

$$
\sum_{x \in T}|\Phi(x)|^{p} \mu(x) \leq C \sum_{z \in T}|\varphi(z)|^{p} 2^{-|z|},
$$

holds for all harmonic functions $\varphi$.

Namely, the requirement that $\varphi$ be harmonic strictly enlarges the set of those $\mu$ for which (97) holds. As mentioned this is analogous to the fact that the condition 83) does not characterizes the class of the Carleson measures for the analytic Hardy space.

Proof of the Corollary. By Theorem 37, the Carleson measures for $H^{2}(T)$ are characterized by (88), which is equivalent to (19) in Section 3. Also, Theorem 40 says that the Carleson measures for $H^{2}(T)$ are exactly those $\varphi$ for which (88) holds whenever $\varphi$ is harmonic. On the other hand, in Section 3, Theorem 11, we showed how to construct measures $\mu$ for which (88) holds, that are not, nonetheless, Carleson measures for $\left(\mathcal{I}, 2,2^{-|z|}\right)$; that is, such that (97) fails for some function.

Proof of the Theorem. We only give an outline of the proof. For $z \in T$, $z \neq o$, let $w_{j}, j=1,2$, be such that $w_{j}>z, d\left(w_{j}, z\right)=1$. define $\varphi_{z}$ as follows

$$
\varphi_{z}(\xi)= \begin{cases}\left(\frac{3}{2}\right)^{1 / 2} 2^{|z| / 2} 2^{-d(z, \zeta)}, & \text { if } \xi \geq w_{1}, \\ -\left(\frac{3}{2}\right)^{1 / 2} 2^{|z| / 2} 2^{-d(z, \zeta)}, & \text { if } \xi \geq w_{2},\end{cases}
$$

and let $\varphi_{z}(\xi)=0$ otherwise. The function $\varphi_{z}$ is harmonic on $T$ and it is, in fact, the Poisson integral of the function $f_{z}: \Omega \longrightarrow \mathbb{R}$ that has values $((3 / 2) 2)^{1 / 2} 2^{|z| / 2}$ on $I\left(w_{1}\right),-(3 / 2)^{1 / 2} 2^{|z| / 2}$ on $I\left(w_{2}\right)$, and 0 otherwise. For $z=o$, we can similarly define two functions $\varphi_{o 1}$ and $\varphi_{o 2}$ in such a way that the set $\left\{\varphi_{z}: z \in T, z \neq o\right\} \cup\left\{\varphi_{o 1}, \varphi_{o 2}, 1\right\}$ is a complete orthonormal system for $H^{2}(T)$. Observe that $1=\mathcal{I} \delta_{o}$, where $\delta_{o}$ is the characteristic function of the set $\{o\}$.

Now let $\Phi \in H^{2}(T)$,

$$
\Phi=a_{0}+\sum_{j=1,2} a_{o j} \mathcal{I} \varphi_{o j}+\sum_{z \in T \backslash\{o\}} a_{z} \mathcal{I} \varphi_{z} .
$$

Then,

$$
\|\Phi\|_{2}^{2} \sim\left|a_{0}\right|^{2}+\sum_{j=1,2}\left|a_{o j}\right|^{2}+\sum_{z \in T \backslash\{o\}}\left|a_{z}\right|^{2}
$$


and $\Phi=\mathcal{I} \varphi$, where

$$
\begin{aligned}
& \|\varphi\|_{L^{2}\left(2^{-|z|}\right)}^{2} \\
& \sim\left|a_{0}\right|^{2}+\sum_{j=1,2}\left|a_{o j}\right|^{2}+\sum_{z \in T \backslash\{o\}}\left|a_{z}\right|^{2} \sum_{j \geq 0} 2^{-(j+|z|)} 2^{j} 2^{2((j+|z|) / 2)} \sim\|\Phi\|_{2}^{2}
\end{aligned}
$$

as claimed.

\section{References.}

[Ad] Adams, D. R., A trace inequality for generalized potentials. Studia Math. 48 (1973), 99-105.

[A] Arcozzi, N., Carleson measures for analytic Besov spaces: the upper triangle case. Preprint, 2000.

[AR] Arcozzi, N., Rochberg, R., Topics in dyadic Dirichlet spaces. Preprint, 2000.

[Bek] Bekollé, D., Inégalités à poids puor le projecteur de Bergman dans la boule unité de $\mathbb{C}^{n}$. Studia Math. 71 (1982), 305-323.

[BB] Bekollé, D., Bonami, A,. Inégalités à poids pour le noyau de Bergman. C. R. Acad. Sci. Paris Sér. A-B 286 (1978), A775-A778.

[Boe] BöE, B., Interpolating Sequences for Besov Spaces. Preprint, 2000.

[Car] Carleson, L., Interpolation by bounded analytic functions and the corona problem. Ann. of Math. 76 (1962), 547-559.

[Cart] Cartier, P., Fonctions harmoniques sur un arbre. Symposia Math. IX, Academic Press, (1972), 203-270.

[CO1] Cascante, C., Ortega, J. M., Carleson measures on spaces of HardySobolev type. Can. J. Math. 47 (1995), 1177-1200.

[CO2] Cascante, C., Ortega, J. M., On $q$-Carleson measures for spaces of $\mathcal{M}$-harmonic functions. Can. J. Math. 49 (1997), 653-674.

[CR] Coifman, R., Rochberg, R., Representation theorems for holomorphic and harmonic functions in $L^{p}$. Astérisque 77 (1980), 11-65.

[DB] Di BiASE, F., Fatou type theorems: maximal functions and approach regions. Birkhäuser, 1997.

[Du] DurretT, R., Brownian motion and martingales in analysis. Wadsworth Inc., 1984.

[EHP] Evans, W. D., Harris, D. J., Pick, L., Weighted Hardy and Poincaré inequalities on trees. J. London Math. Soc. 52 (1995), 121-136. 
[KS] Kerman, R., Sawyer, E., Carleson measures and multipliers of Dirichlet-type spaces. Trans. Amer. Math. Soc. 309 (1988), 87-98.

[KPt] Korányi, A., Picardello, M., Taibleson, M., Hardy spaces on nonhomogeneous trees. With an appendix by Picardello and Wolfgang Woess. Symposia Mathematica XXIX (Cortona, 1984), 205-265, Academic Press, 1987.

[L] LueCKing, D., Representation and duality in weighted spaces of analytic functions. Indiana Univ. J. 34 (1985), 319-336.

[L2] LEUCKING, D., Embedding theorems for spaces of analytic functions via Kinchine's inequality. Michigan Math. J. 40 (1993), 333-358.

[MS] Marshall, D. E., Sundberg, C., Interpolating sequences for the multipliers of the Dirichlet space. Preprint.

[Muck] Muckenhoupt, B., Weighted norm inequalities for classical operators. Proc. Symp. in Pure Math. XXXV Amer. Math. Soc. (1979), 69-84.

[R] Rochberg, R., Interpolating by functions in Bergman spaces. Mich. Math. J. 29 (1982), 229-236.

[RW] Rochberg, R., Wu, Z., A new characterization of Dirichlet type spaces and applications. Illinois J. Math. 37 (1993), 101-122.

[Saw] SAwYER, E., Weighted inequalities for the two-dimensional Hardy operator. Studia Math. LXXXII (1985), 1-15.

[Saw2] SAwYer, E., A two weight weak type inequality for fractional integrals. Trans. Amer. Math. Soc. 281 (1984), 339-345.

[SW] SAWyer, E., Wheeden, R. L., Weighted inequalities for fractional integrals on Euclidean and homogeneous spaces. Amer. J. of Math. 114 (1992), 813-874.

[So] SoARdi, P. M., Potential theory on infinite networks. Lecture Notes in Math. 1590, Springer, 1994.

[Ste] Stegenga, D. A., Multipliers of the Dirichlet space. Illinois J. Math. 24 (1980), 113-139.

[St] SteIn, E., Singular integrals and differentiability properties of functions. Princeton Univesity Press, 1970.

[T] Taibleson, M., Hardy spaces of harmonic functions on homogeneous isotropic trees. Math. Nachr. 133 (1987), 273-288.

[Ve] Verbitsky, I. È., Multipliers in spaces with "fractional" norms, and inner functions. (Russian) Sibirsk. Mat. Zh. 26 (1985), 51-72, 221. Translated in Siber. J . of Mathematics 26 (1985), 198-216.

[Wang] Wang, J., Ph. D. Thesis. Washington University in St. Louis, 1995. 
[Wu $\mathrm{Wu}, \mathrm{Z}$., Carleson measures and multipliers for Dirichlet spaces. J. Funct. Anal. 169 (1999), 148-163.

[Zhu] ZHU, K., Operator theory on function spaces. Marcel Dekker Inc., 1990.

Recibido: 26 de enero de 2000

Nicola Arcozzi*

Dipartimento di Matematica

Universita di Bologna

Piazza di Porta San Donato, 5

40127, Bologna, ITALY

arcozzi@dm.unibo.it

Richard Rochberg ${ }^{\dagger}$

Washington University

Department of Mathematics

Cupples I, Room 100

Campus Box 1146

St. Louis, MO 63130-4899, U.S.A.

rr@wustl.edu

Eric Sawyer $\ddagger$

Department of Mathematics \& Statistics

Burke Science Building Rm. 133

McMaster University 1280 Main Street West

Hamilton, Ontario, L8S 4K1, CANADA

sawyer@mcmail.cis.mcmaster.ca

* Partially supported by a grant of the Italian Minister of University and Research.

$\dagger$ Supported in part by a grant from the National Science Foundation.

$\ddagger$ Supported in part by a grant from the National Science Foundation. 